\title{
Direct Numerical Simulation of Supersonic Flow and Acoustics over a Compression Ramp
}

\author{
loannis W. Kokkinakis, ${ }^{1,}$ a) Dimitris Drikakis, ${ }^{2, \text { b) }}$ Konstantinos Ritos, ${ }^{1, c)}$ and S. Michael Spottswood ${ }^{3}$ \\ ${ }^{1)}$ Department of Mechanical \& Aerospace Engineering, University of Strathclyde, Glasgow, G1 1XJ, UK \\ 2) University of Nicosia, Nicosia CY-2417, Cyprus \\ ${ }^{3)}$ Air Force Research Laboratory, Wright Patterson AFB, Ohio 45433-7402, USA
}

(Dated: 4 June 2020)

We present Direct Numerical Simulations (DNS) of shock turbulent boundary layers interaction (SBLI) at Mach 2.9 over a $24^{\circ}$ ramp. We study both the numerical accuracy and flow physics. Two classes of spatial reconstruction schemes are employed: the Monotonic Up-stream-centred Scheme for Conservation Laws (MUSCL) and the Weighted Essentially Non-Oscillatory (WENO), of accuracy ranging from $2^{\text {nd }}$ to $11^{\text {th }}$-order. Using the canonical Taylor-Green vortex (TGV) test-case, a simple and computationally inexpensive rescaling of the candidate stencil values - within the context of the high-order WENO scheme - is proposed for reducing the numerical dissipation, particularly in underresolved simulations. For the compression ramp case, higher-order schemes are shown to capture the size of the SBLI separation zone more accurately, a consequence of resolving much finer turbulence structures. For second and fifthorder schemes, the energy of the unresolved small scale turbulence shifts the cascade of the turbulence kinetic energy spectrum, thus resulting in more energetic large scale turbulent structures. Consequently, the $\lambda$-shock foot shifts further downstream leading to a smaller separation bubble size. Nonetheless, other statistical quantities, such as the turbulence anisotropy invariant map and the turbulence kinetic energy budget terms, show little dependence on the type and order of the spatial reconstruction scheme. Finally, using the more accurate $9^{\text {th }}$-order WENO results, it is reasoned that the interaction of the $\lambda$-shock with the post-shock relaxation region drives the low-frequency oscillation of the $\lambda$-shock.

\section{INTRODUCTION}

Design processes in complex engineering systems require satisfying various degrees of constraints to adhere to design quality standards. Through careful planning and availability of instrumentation or apparatus that conforms to the necessary specifications, experimental results can assist the design of engineering systems considerably. However, most experiments are deemed cumbersome and require considerable time to plan, execute and later analyze. The above is particularly true when a rigorous and arduous design cycle or an optimization approach is needed early on in the design phase. Furthermore, the availability of diagnostic instrumentation limits the amount of useful information, which can be obtained from the experiments.

Computational methods offer the opportunity to obtain a high turnover of results along with ample amounts of data, typically within a relatively short time, allowing for a plethora of key design parameters to be altered and assessed during the initial development phase of a product. Computational methods have become increasingly more popular and widely used, particularly at the early design phase of many engineering applications, particularly those problems where experimental data are scarce. However, the solutions provided can contain significant inaccuracies. The uncertainties in computational methods are associated with several assumptions regarding turbulence modelling and the excessive numerical dissipation

a)Electronic mail: ioannis.kokkinakis@strath.ac.uk

${ }^{b)}$ Electronic mail: Author to whom correspondence should be addressed drikakis.d@unic.ac.cy. Also affiliated with the University of Nicosia Research Foundation and Defence and Security Research Institute.

c)Electronic mail: konstantinos.ritos@strath.ac.uk of discretization methods mainly when simulations are performed on coarse grids. With the availability of ever more increasing computational power, high-order large eddy simulation (LES) has gradually started to become more feasible and widely adopted in industrial applications. Furthermore, highresolution and high-order methods for compressible flows featuring shock-waves and turbulence allow performing both implicit Large Eddy Simulations (iLES) and DNS in the same computational framework ${ }^{1-3}$.

A common phenomenon prevalent in high-speed flows around aircraft is shock-wave boundary layer interaction (SBLI). Typically, SBLI occurs wherever the surface of an aerodynamic vehicle, travelling faster than the (free-stream) speed of sound, protrudes abruptly towards the flow, as in the case of a deflected flap or an inlet ramp. The ensuing complex phenomena - including flow separation and re-attachment, low-frequency unsteadiness and high local thermal loads - can significantly affect the aerodynamic performance and structure of a supersonic vehicle. As a consequence, such phenomena and interactions have been extensively - and still are - actively investigated, both experimentally $y^{4-11}$ as well as numerically ${ }^{12-27}$.

Provided that the shock-wave is sufficiently strong, a recirculation bubble in the boundary layer will appear where the shock impinges to the surface of the aero-structure. Observations indicate that the shock-bubble interaction can oscillate with a characteristic frequency of one or two orders of magnitude lower than the typical frequency of the supersonic turbulent boundary layer (TBL), and with a length scale much larger than the TBL thickness. The oscillation frequency is often close to the structural resonance frequencies of the aerostructure. Thus, it is essential to predict it accurately. Researchers have directed their efforts towards the understanding of the oscillatory behaviour of the separation shock-bubble 
system $^{22,27-30}$. Yet, the physical mechanism behind the lowfrequency unsteadiness still remains elusive to-date and is widely debated. The current literature suggests that its origin is dependent on the flow properties, either upstream or downstream of the interaction. Clemens and Narayanaswamy ${ }^{29}$ have given an extensive review on this topic. The aerostructural response of structures at supersonic and hypersonic conditions subjected to acoustic fatigue due to pressure fluctuations beneath TBLs and SBLI has been the subject of recent experimental studies ${ }^{31,32}$, as well as the present numerical investigation.

We have employed shock-capturing, high-order schemes with nominal accuracy up to $9^{\text {th }}$-order, which has previously used in wall-resolved implicit Large Eddy Simulations ${ }^{33-35}$ of compressible turbulent boundary layers, amongst other flows, and perform DNS of SBLI. Our motivation is to investigate the effects of numerical dissipation on numerical accuracy in the case of compressible DNS. We perform simulations for an incoming fully-turbulent boundary-layer at a freestream Mach number of 2.9 that forms an unsteady $\lambda$-shock wave structure at the corner of a $24^{\circ}$ compression ramp. The use of a synthetic turbulence inflow allows for a smaller domain in the streamwise direction. The results show that high-order DNS methods are particularly well suited for simulating SBLI and prove resilient to the excess numerical dissipation of loworder reconstruction schemes. We show that the lower order schemes under-predict the skin-friction coefficient before the SBLI, as well as the size of the separation bubble. Using low-Mach correction ${ }^{36}$ with the MUSCL-type schemes is found to offer slight improvement to the near-wall resolution of the TBL and size of the separation bubble. For a freestream Mach number of 2.9, the subsonic region of the "undisturbed" (pre-SBLI) TBL is found to mostly reside within the viscoussublayer and lower buffer-layer $\left(y^{+}<12\right)$, which is "energized" by the frequent "injections" of turbulence generated in the supersonic part of the TBL.

WENO schemes have been extensively used in the past to increase the accuracy of compressible flow simulations ${ }^{37-40}$. We propose a low-cost and straightforward normalization technique of the WENO computational stencil values to improve accuracy and stability. We use the canonical TaylorGreen vortex (TGV) problem to show that the pre-treatment of the WENO stencils helps to further reduce its numerical dissipation, particularly for coarser meshes. A physical mechanism for the low-frequency oscillation of the oblique shock wave is also proposed. We found that there is pressuredriven reciprocity between the separated (free-shear) layer reattachment and separation bubble (edge locations), and the acoustic propagation speed matches that of the measured lowfrequency oscillation.

\section{GOVERNING EQUATIONS}

The compressible Navier-Stokes equations (NSE) are solved for an ideal gas using the finite volume method (FVM).
In integral form, the NSE are formulated as:

$$
\begin{gathered}
\frac{\partial}{\partial t} \iiint_{V} \rho \mathrm{dV}=-\oiint_{A} \rho(\mathbf{u} \cdot \hat{\mathbf{n}}) \mathrm{dA} \\
\frac{\partial}{\partial t} \iiint_{V} \rho \mathbf{u} \mathrm{dV}=-\oiint_{A} \rho \mathbf{u}(\mathbf{u} \cdot \hat{\mathbf{n}}) \mathrm{dA}-\oiint_{A} p \hat{\mathbf{n}} \mathrm{dA} \\
+\oiint_{A}(\tau \cdot \hat{\mathbf{n}}) \mathrm{dA} \\
\frac{\partial}{\partial t} \iiint_{V} \rho e \mathrm{dV}=-\oiint_{A} \rho(\mathbf{u} \cdot \hat{\mathbf{n}}) e \mathrm{dA}-\oiint_{A} p(\mathbf{u} \cdot \hat{\mathbf{n}}) \mathrm{dA} \\
+\oiint_{A}(\mathbf{u} \cdot \tau) \cdot \hat{\mathbf{n}} \mathrm{dA}-\oiint_{A}(\mathbf{q} \cdot \hat{\mathbf{n}}) \mathrm{dA}
\end{gathered}
$$

where $\rho$ is the density; $\mathbf{u}$ is the velocity vector; $p$ is the static pressure; $\hat{\mathbf{n}}$ is the outward pointing unit normal of surface element $\mathrm{dA}$ of the closed finite control volume $\mathrm{dV} ; e$ is the total energy per unit mass given by $e=i+\mathbf{u} \cdot \mathbf{u} / 2 ; i$ is the specific internal energy, which for a calorically perfect gas is given by:

$$
i=c_{v} T=\frac{p}{\rho(\gamma-1)}
$$

$T$ is the temperature, $c_{v}$ is the specific heat capacity at constant volume, and $\gamma$ is the heat capacity ratio (or adiabatic index) defined as $\gamma=c_{p} / c_{v}$ where $c_{p}$ is the specific heat capacity at constant pressure and $R_{s}=c_{p}-c_{v}=c_{v}(\gamma-1)$ is the specific gas constant.

For a Newtonian fluid, the shear stress tensor is given by:

$$
\tau=\lambda_{b}(\nabla \cdot \mathbf{u}) I+\mu\left[\nabla \mathbf{u}+(\nabla \mathbf{u})^{T}\right]
$$

where I is the identity tensor; the bulk viscosity is given by $\lambda_{b}=-4 \mu / 3$ according to Stoke's hypothesis; and $\mu$ is the dynamic viscosity obtained by Sutherland's Law as:

$$
\mu(T)=\mu_{r e f}\left(\frac{T}{T_{r e f}}\right)^{\frac{3}{2}} \frac{T_{r e f}+T_{s}}{T+T_{s}}
$$

where the free-stream values are used as the reference and the Sutherland temperature is $T_{s}=110.4 \mathrm{~K}$.

The heat flux is calculated by Fourier's Law of heat conduction:

$$
\mathbf{q}=-\kappa \nabla T
$$

where $\kappa$ is the heat conductivity given by:

$$
\kappa(T)=\frac{c_{p}}{\operatorname{Pr}} \mu(T)
$$

and $\operatorname{Pr}=0.72$ is Prandtl's number. 


\section{NUMERICAL METHODS}

We employ the block-structured grid code CNS3D that solves the Navier-Stokes equations using the finite-volume method (FVM). The advective terms are solved using the Godunov-type (upwind) method, whose inter-cell numerical fluxes are calculated by solving the Riemann problem using the reconstructed values of the primitive variables at the cell interfaces. A one-dimensional swept unidirectional stencil is used for the spatial reconstruction. Two different slope limiting approaches have been implemented in conjunction with the approximate HLLC Riemann solver, namely the: (i) Monotone upstream-centred schemes for conservation laws (MUSCL), and (ii) Weighted essentially non-oscillatory (WENO). In particular, the following slope-limiters are examined:

- MUSCL $2^{\text {nd }}$ order Monotonized Central (M2) ${ }^{41}$;

- MUSCL $5^{\text {th }}$ (M5) order ${ }^{42}$;

- WENO $9^{\text {th }}\left(\right.$ W9) order $^{39}$.

Finally, we use a second-order central scheme for the viscous terms. The solution is advanced in time by using a five-stage (fourth-order accurate) optimal strong-stabilitypreserving Runge-Kutta method ${ }^{43}$. Further details of the numerical aspects of the code can be found in ${ }^{44,45}$ and references therein.

\section{A. Low Mach number correction}

The accuracy of the above schemes, as well as of any other, can be further enhanced in low-speed subsonic conditions by implementing the low-Mach correction of Thornber et al. ${ }^{36}$ (henceforth labelled LMC). The low-Mach correction primarily involves an additional computational step that treats the velocity vector via a progressive central differencing of its components. The LMC ensures a balanced distribution of dissipation of kinetic energy in the limit of zero Mach number, thus extending the validity of compressible flow codes to Mach numbers as low as $10^{-5}$, and is mainly required for schemes providing accuracy less than $5^{\text {th }}$-order ${ }^{44,45}$.

After the reconstruction of the velocities has been carried out, the reconstructed left and right velocity components at cell-face $(i+1 / 2)$ are modified according to:

$$
\begin{aligned}
\mathbf{u}_{i+1 / 2}^{L, \text { new }} & =\left(\mathbf{u}_{s}-\mathbf{u}_{u}\right) / 2 \\
\mathbf{u}_{i+1 / 2}^{\text {Rnew }} & =\left(\mathbf{u}_{s}+\mathbf{u}_{u}\right) / 2
\end{aligned}
$$

where

$$
\begin{aligned}
\mathbf{u}_{s} & =\mathbf{u}_{i+1 / 2}^{L}+\mathbf{u}_{i+1 / 2}^{R} \\
\mathbf{u}_{u} & =\left(\mathbf{u}_{i+1 / 2}^{L}-\mathbf{u}_{i+1 / 2}^{R}\right) M_{\max }
\end{aligned}
$$

and the maximum local Mach number, $M_{\max }$, is given by:

$$
M_{\max }=\max \left(M_{i+1 / 2}^{L}, M_{i+1 / 2}^{R}\right)
$$

Note that the density and pressure are not altered in any way during this step, thus the internal energy component ( $\rho i=$ $p /(\gamma-1))$ remains unchanged. The reconstructed left and right total energies $\left(e=i+e_{k}\right)$ are calculated using the modified velocities in the kinetic energy component $\left(e_{k}=\mathbf{u} \cdot \mathbf{u} / 2\right)$.

\section{B. WENO implementation}

To address potential numerical instabilities due to the process of choosing an essentially non-oscillatory (ENO) stencil ${ }^{46}$, Weighted ENO (WENO) methods were introduced $^{37,47}$. WENO schemes use a convex combination of all the ENO candidate stencils such that the numerical flux is approximated with the higher order of accuracy in smooth regions, while still retaining the ENO property in the flow regions near discontinuities; see $\mathrm{s}^{48,49}$ for an overview and further references. For WENO implementations on structured grids, when the solution is locally smooth enough, the convex combination of the stencils of a $r^{\text {th }}$-order ENO scheme results in a $(2 r-1)^{\text {th }}$-order WENO scheme ${ }^{47}$.

Aiming to achieve a balance between accuracy and stability, we enhance the WENO schemes of $3^{\text {rd }}$ and $5^{\text {th }}$-order of Jiang and $\mathrm{Shu}^{37}(r=2,3)$ and $7^{\text {th }}, 9^{\text {th }}$ and $11^{\text {th }}$-order of Balsara and $\mathrm{Shu}^{39}(r=4,5,6)$ by combining the mapped WENO approach of Henrick, Aslam, and Powers ${ }^{38}$ (WENO-M) and the relative total variation limiter approach of Taylor, $\mathrm{Wu}$, and Martín ${ }^{40}$ (WENO-RLTV). WENO-M recovers the loss of accuracy occurring near smooth critical points. WENORLTV reduces the numerical dissipation by using the optimal linear weights in regions sufficiently smooth instead of the nonlinear smoothness-indicator-based weights. The numerical reconstruction can be performed at the level of conservative, characteristic, or primitive variables. The reconstruction of the conservative variables is more common in the literature. However, past research has shown that such practice can lead to inaccuracies in capturing shock waves; see Zanotti and Dumbser ${ }^{50}$ and references therein. Similar to other authors ${ }^{51}$, we have opted to use the primitive variables in the high-order numerical reconstruction. The characteristics-based variables would be more expensive computationally.

We present below a step-by-step description of the WENO procedure implemented to obtain the left reconstruction, $q_{i+1 / 2}^{L}$, of the primitive variables, $q=[\rho, \mathbf{u}, p]^{T}$, at cell face $i+1 / 2$ :

1. The full (left and right reconstruction) stencil $\left(S_{i+1 / 2}^{G}\right)$ is normalized, per variable, according to the transformation function:

$$
S_{i+1 / 2}^{G z}=\frac{S_{i+1 / 2}^{G}-g_{\min }}{g_{\max }}
$$

where

$$
S_{i+1 / 2}^{G}=\left(q_{i-r+1}, \ldots, q_{i+r}\right)
$$


and

$$
\begin{aligned}
& g_{\min }=\min \left(S_{i+1 / 2}^{G}\right)-1 \\
& g_{\max }=\max \left(S_{i+1 / 2}^{G}-q_{\min }\right)
\end{aligned}
$$

and the new $k^{\text {th }}$ candidate stencil for the left reconstruction, containing $r$ cell center values, is given by:

$$
S_{i+1 / 2 ; k}^{L}=S_{i+1 / 2}^{G z}[i-r+1+k, \ldots, i+k]
$$

where $k=0, \ldots, r-1$. Eq. (7) simply normalizes the values of the candidate stencils prior to the estimation of the smoothness indicators (IS) in such a way that (i) the maximum value of the full stencil becomes equal to one, i.e. $\max \left(S_{i+1 / 2}^{G z}\right)=1$, (ii) the minimum value takes a positive and nonzero value, i.e. $\min \left(S_{i+1 / 2}^{G z}\right)>0$, and (iii) the value range scales as originally relative to the maximum. By definition $g_{\max }$ is always positive and non-zero and hence Eq. (7) will never result in an undefined operation and cause an exception. Using the above normalization of the full stencil values, per variable, is found to (i) prevent negative WENO smoothness indicator values, (ii) reduce numerical dissipation, and (iii) simplify the application of the proceeding step. The stencil normalization was found to have no effect for the MUSCL-type slope limiters.

2. Next, a modified version of the relative total variation (TV) limiting procedure of Taylor, Wu, and Martín ${ }^{40}$ is implemented. The TV of each $k^{\text {th }}$ candidate stencil is calculated according to:

$$
\operatorname{TV}_{k}\left(S_{i ; k}\right)=\sum_{l=1}^{r-1}\left|q_{i-r+k+l+1}-q_{i-r+k+l}\right|
$$

Eq. (8) is then used to obtain the maximum TV ratio between the candidate stencils:

$$
R(\mathrm{TV})=\frac{\max \left(\mathrm{TV}_{k}\right)}{\min \left(\mathrm{TV}_{k}\right)+\varepsilon}
$$

If all of the stencils contain significant discontinuities, then the value of $R(\mathrm{TV})$ can be incorrectly small, i.e. $R(\mathrm{TV}) \approx 1$. Thus, an additional criteria is introduced in order to avoid such a situation. The linear weights are used provided the following two conditions are satisfied:

$$
\begin{gathered}
\text { if }\left[R(\mathrm{TV})<A_{\mathrm{RL}}^{\mathrm{TV}} \&\right. \\
\left.\max \left(\mathrm{TV}_{k}\right)<B_{\mathrm{RL}}^{\mathrm{TV}}\right] \text { then } \\
\omega_{k}^{r}=C_{k}^{r}
\end{gathered}
$$

According to Ref. ${ }^{40}, A_{\mathrm{RL}}^{\mathrm{TV}}=5$, while for the second condition, $B_{\mathrm{RL}}^{\mathrm{TV}}=0.2(r-1)$, where $r$ is the order of the polynomials used in the $2(r-1)^{\text {th }}$-order WENO. Note that the equation for $B_{\mathrm{RL}}^{\mathrm{TV}}$ is applicable only if the preceding pre-treatment/re-scaling of the candidate stencils is carried out; otherwise it must be multiplied by $q_{\max }$. In essence, the second condition allows for an average TV of $20 \%$ between two neighbouring cells of the local stencils $\left(S_{i}^{G}\right)$ maximum variable value, but this value can be modified if necessary. $\mathrm{Wu}$ and Martin ${ }^{16}$ used a value of $B_{\mathrm{RL}}^{\mathrm{TV}}=0.2$ for their $4^{\text {th }}$-order bandwidthoptimized WENO implementation in their DNS study.

Eq. (10) assumes that for the linear weights the condition $\sum_{l=0}^{r-1} C_{l}^{r}=1$ is always satisfied.

3. If condition Eq. (10) is not satisfied, then the nonlinear weights based on the smoothness indicators of each candidate stencil are computed according to the following two steps:

$$
\Omega_{k}^{r}=\frac{C_{k}^{r}}{\left(\mathrm{IS}_{k}^{r}\right)^{p}+\varepsilon}, \quad \omega_{k}^{r}=\frac{\Omega_{k}^{r}}{\sum_{l=0}^{r-1} \Omega_{l}^{r}}
$$

where $p=r$ and $\varepsilon=10^{-41}$.

The standard WENO weights obtained in Eq. (11) are modified according to the mapped WENO (WENO-M) approach of Henrick, Aslam, and Powers ${ }^{38}$ as:

$$
\tilde{\omega}_{k}^{r}=\frac{\tilde{\Omega}_{k}^{r}}{\sum_{l=0}^{r-1} \tilde{\Omega}_{l}^{r}}
$$

where, using the alternate formulation of Feng, Huang, and Wang ${ }^{52}$, the mapped weights are given by:

$$
\tilde{\Omega}_{k}^{r}=C_{k}^{r}+\frac{\left(\Omega_{k}^{r}+C_{k}^{r}\right)^{K+1} A}{\left(\Omega_{k}^{r}-C_{k}^{r}\right)^{K} A+\Omega_{k}^{r}\left(1-\Omega_{k}^{r}\right)}
$$

and setting $A=1$ and $K=2$ results in the original mapping function ${ }^{38}$.

4. The reconstructed scaled variable value at the left-side of cell-face $i+1 / 2$ is given by:

$$
q_{i+1 / 2}^{L}=\sum_{k=0}^{r-1}\left[\tilde{\omega}_{k}^{r} f(q)_{k}^{r}\right]
$$

where

$$
f(q)_{k}^{r}=\sum_{l=0}^{r-1} \alpha_{k ; l}^{r} q_{i-r+k+l+1}
$$

5. Finally, due to the initial "normalizing" of the stencil in step 1, the reconstructed values obtained using Eq. (14) needs to be "re-scaled" according to:

$$
q_{i+1 / 2}^{L}=q_{i+1 / 2}^{L} g_{\max }+g_{\min }
$$

WENO reconstruction can lead to spurious oscillations, if two or more shocks are too close to each other and WENO cannot choose a single smooth stencil. To remedy this problem a procedure first introduced by Harten et al. ${ }^{53}$ is adopted. 
If the reconstructed density and pressure values differ too drastically from their cell-center average values, the order of the WENO reconstruction is reduced. After completion of the left and right reconstruction procedures at cell-face $i+1 / 2$, the left and right reconstructed density and pressure values are compared against their respective left and right cell-center values:

$$
\begin{aligned}
\left|\rho_{i+1 / 2}^{L}-\rho_{i}\right| & >\mathrm{C}_{\mathscr{O}}^{-} \quad \text { or } \\
\left|\rho_{i+1 / 2}^{R}-\rho_{i+1}\right| & >\mathrm{C}_{\mathscr{O}}^{-}
\end{aligned}
$$

where the order reduction threshold constant is set equal to $\mathrm{C}_{\mathscr{O}}^{-}=0.7$. If the condition in Eq. (17) is met, then the order of the WENO scheme is reduced according to $(r-1)$. The reconstruction procedure is then repeated for all variables, and the condition is checked again. The process is repeated until Eq. (17) is not satisfied any longer. For example, assuming the condition is repeatably met, a $9^{\text {th }}$-order WENO would first reduce to $7^{\text {th }}$-order, then to $5^{\text {th }}, 3^{\text {rd }}$, and finally to the $2^{\text {nd }}$-order MC MUSCL scheme. Titarev and Toro ${ }^{54}$ showed that the use of the above procedure does not degrade the high order of accuracy for sufficiently smooth solutions.

\section{HLLC Riemann solver implementation}

The Riemann problem is solved here using the so-called "Harten, Lax, van Leer, and (the missing) Contact" (HLLC) approximate Riemann solver of Toro, Spruce, and Speares ${ }^{55}$. More specifically, the adaptive non-iterative Riemann solver (ANRS) variant proposed by Toro ${ }^{56}$ (see $\$ 9.5 .2$ ) is implemented. The following sequence details the approximate HLLC Riemann solver procedure implemented:

1. To ensure high-order near the boundaries for high-order FVM codes, typically the ghost-cell method is used to apply the boundary conditions (BC). However, even after careful programming of the boundary conditions and reconstruction procedures, computer rounding errors can persist and give rise to differences between the left and right reconstructed states. Therefore, to ensure the appropriate flux, we modify the left and right reconstructed states for the following BCs: symmetry plane (inviscid wall), heated (constant temperature) wall, and adiabatic (zero heat-flux) viscous (no-slip) wall.

For a symmetry plane, the no penetration condition is implemented for both advective and acoustic waves using the procedure described by Algorithm 1.

In the case of a viscous wall, Algorithm 2 is used instead. For an isothermal wall, the temperature at the ghost cells is linearly interpolated from the interior domain and the wall. In this case, it is advisable to restrict the interpolated temperature range of values to be only positive $\left(T \in \mathbb{R}_{>0}\right)$, i.e. $T_{\text {ghosts }}>10^{-15}$, which reduces the likelihood of a non-physical solution from manifesting. if Left BC Symmetry then

$$
\begin{aligned}
& \rho_{L}=\rho_{R} ; \\
& p_{L}=p_{R} ; \\
& \mathbf{u}_{R}=\mathbf{u}_{R}-\left(\mathbf{u}_{R} \cdot \hat{\mathbf{n}}\right) \mathbf{u}_{R} ; \\
& \mathbf{u}_{L}=\mathbf{u}_{R} ;
\end{aligned}
$$

else if Right $B C$ Symmetry then

$$
\begin{aligned}
& \rho_{R}=\rho_{L} ; \\
& p_{R}=p_{L} ; \\
& \mathbf{u}_{L}=\mathbf{u}_{L}-\left(\mathbf{u}_{L} \cdot \hat{\mathbf{n}}\right) \mathbf{u}_{L} ; \\
& \mathbf{u}_{R}=\mathbf{u}_{L} ;
\end{aligned}
$$

Algorithm 1: Ensure symmetry BC flux in HLLC.

if Left BC Viscous Wall then

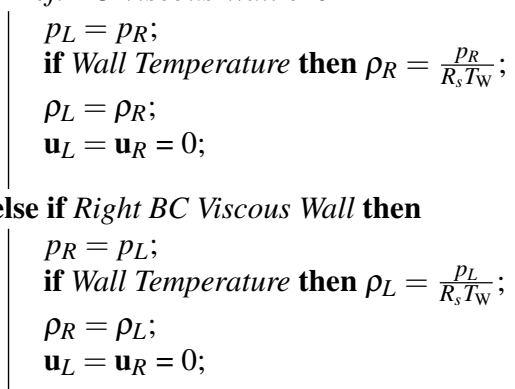

Algorithm 2: Ensure viscous wall BC flux in HLLC; if Wall Temperature true isothermal, else adiabatic.

2. An initial estimate of the pressure in the Star Region, that is the region defined in-between the two non-linear convective wave-speeds (or characteristics), can be obtained according to ${ }^{57}$ :

$$
p_{*}=\max \left(0, p_{\text {pvrs }}\right)
$$

which for curvilinear coordinates $p_{\text {pvrs }}$ is obtained according to:

$$
\begin{aligned}
p_{\mathrm{pvrs}} & =\frac{1}{2}\left[p_{L}+p_{R}+\left(u_{L}^{\perp}-u_{R}^{\perp}\right) \bar{\rho} \bar{s}\right] \\
\bar{\rho} & =\left(\rho_{L}+\rho_{R}\right) / 2, \quad \bar{s}=\left(s_{L}+s_{R}\right) / 2
\end{aligned}
$$

where the speed of sound is defined as $s=\sqrt{\gamma p / \rho}$ and $u^{\perp}=\mathbf{u} \cdot \hat{\mathbf{n}}$ is the magnitude of the velocity normal to the cell-face.

The "averaged" value of $p_{*}$ given by Eq. (18), is enhanced by taking into account the local conditions. The ANRS approach ${ }^{56}$ introduces two conditions as a means to avoid unnecessary computations, i.e. updating the value of $p_{*}$ obtained by Eq. (18) with one that is more accurate. The first condition requires that the ratio between the maximum and minimum local reconstructed pressures is greater than a predetermined constant, i.e.

$$
Q=p_{\max } / p_{\min }>Q_{\text {user }}
$$

where $p_{\min }=\min \left(p_{L}, p_{R}\right), p_{\max }=\max \left(p_{L}, p_{R}\right)$ and it is recommended that $Q_{\text {user }}=2$. The other condition requires that $p_{*}$ does not lie between $p_{\min }$ and $p_{\max }$, 
i.e. $p_{*}<p_{\min }$ or $p_{*}>p_{\max }$. However, likewise to nondifferentiable (reconstruction) limiters, they hinder convergence. Instead, the following relation is used immediately after Eq. (18):

$p_{*}= \begin{cases}{\left[\frac{s_{L}+s_{R}-\frac{\gamma-1}{2}\left(u_{R}^{\perp}-u_{L}^{\perp}\right)}{s_{L} / p_{L}^{z}+s_{R} / p_{R}^{z}}\right]^{\frac{1}{z}}} & \text { if } p_{*} \stackrel{\text { (TRRS) }}{\leq} p_{\min } \\ \frac{g_{L} p_{L}+g_{R} p_{R}-\left(u_{R}^{\perp}-u_{L}^{\perp}\right)}{g_{L}+g_{R}} & \text { if } p_{*}{ }^{\text {(TSRS) }}>p_{\max }\end{cases}$

where

$$
\begin{gathered}
z=\frac{\gamma-1}{2 \gamma}, \quad A_{K}=\frac{2}{(\gamma-1) \rho_{K}}, \quad B=\left(\frac{\gamma-1}{\gamma+1}\right) p_{K} \\
g_{K}=\left(\frac{A_{K}}{p+B_{K}}\right)^{1 / 2}, \quad K=L, R
\end{gathered}
$$

The abbreviations TRRS ${ }^{56}$ and TSRS ${ }^{56,58}$ stand for the Two-Rarefaction Riemann Solver and Two-Shock Riemann Solver, respectively.

3. Next, we compute the wave-speed estimates according to a modified formulation which combines the approaches of Davis ${ }^{59}$ and Toro, Spruce, and Speares ${ }^{55}$ :

$$
\begin{array}{r}
S_{L}=\min \left(u_{L}^{\perp}-s_{L} q_{L}, u_{R}^{\perp}-s_{R} q_{R}\right) \\
S_{R}=\max \left(u_{L}^{\perp}+s_{L} q_{L}, u_{R}^{\perp}+s_{R} q_{R}\right)
\end{array}
$$

where

$q_{K}= \begin{cases}1 & \text { if } p_{*} \leq p_{K} \\ {\left[1+\frac{\gamma+1}{2 \gamma}\left(\frac{p_{*}}{p_{K}}-1\right)\right]^{1 / 2}} & \text { if } p_{*}>p_{K}\end{cases}$

Eq. (20) slightly increases the numerical diffusion as it permits a greater range, but has favourable stability in very high-speed flows and particularly near strong shock-waves.

Using the above, the intermediate "missing" wave-speed, $S_{*}$, and associated HLLC fluxes are computed according to Toro $^{56}$.

\section{Taylor-Green Vortex}

The Taylor-Green vortex (TGV) is a well-known and widely used benchmark test-case used by the wider computational fluid dynamics community to assess the properties of numerical schemes. Figures 1(a) and 1(b) show the kinetic energy dissipation obtained using the described WENO numerical procedure without and with the stencil normalization for various grid resolutions, respectively. We compare the present DNS, performed on $256^{3}$ mesh, with the DNS of DeBonis ${ }^{60}$, which was conducted using a finer mesh $\left(512^{3}\right)$ and the 13point dispersion-relation-preserving (DRP) scheme of Bogey

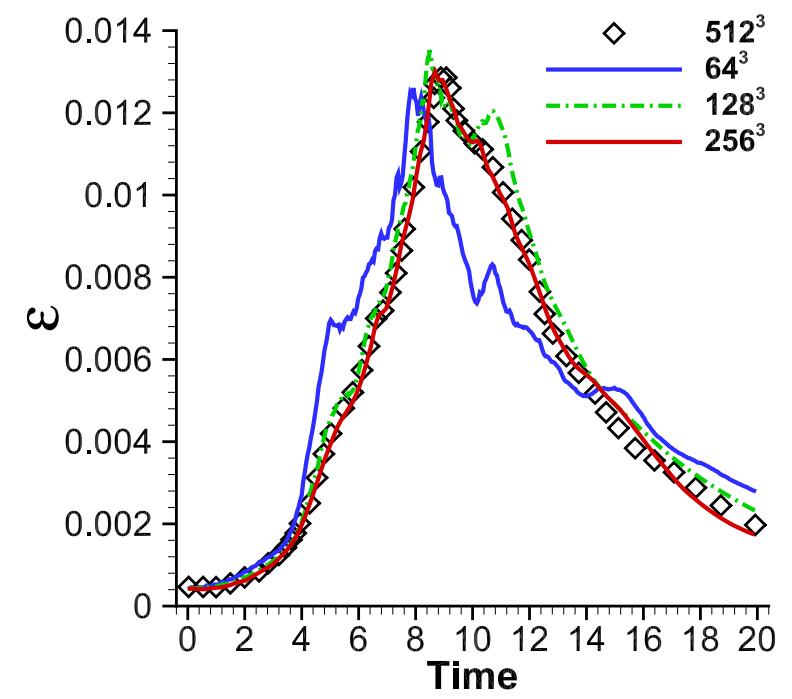

(a)

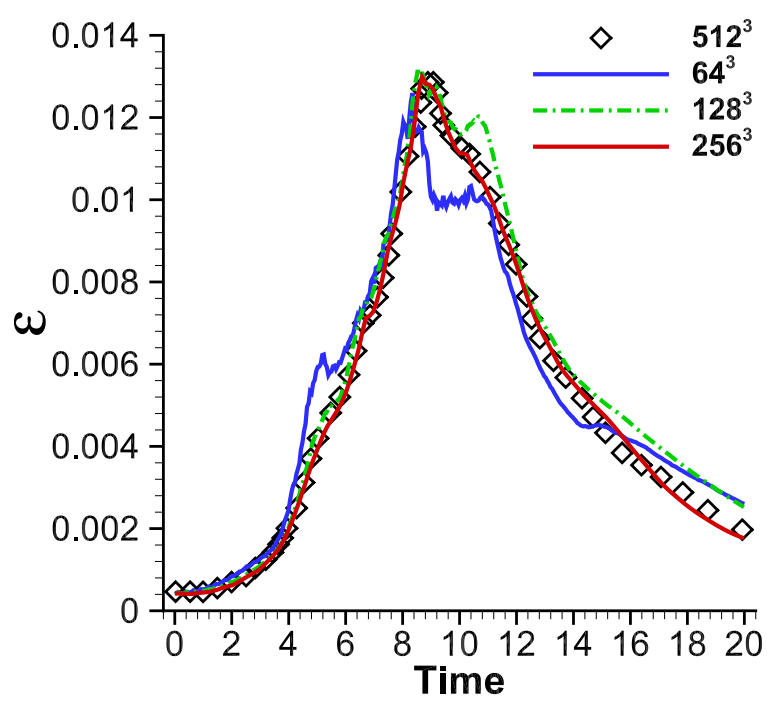

(b)

FIG. 1. Comparison of the turbulence kinetic energy dissipation $(\varepsilon)$ for the Taylor-Green vortex test case using the $11^{\text {th }}$-order WENO $(r=6)$ (a) without, and (b) with the stencil normalization.

and Bailly ${ }^{61}$. We calculate the energy and dissipation as follows:

$$
\begin{gathered}
E_{k}=\frac{1}{\rho_{0} V} \int \frac{1}{2} \rho(\mathbf{u} \cdot \mathbf{u}) \mathrm{dV}, \quad \varepsilon_{2}=-\frac{1}{\rho_{0} V} \int p(\nabla \cdot \mathbf{u}) \mathrm{dV} \\
\varepsilon_{1}=-\frac{d E_{k}}{d t}, \quad \varepsilon=\varepsilon_{1}-\varepsilon_{2}
\end{gathered}
$$

The $11^{\text {th }}$-order WENO implementation with stencil normalization significantly reduces dissipation $(\varepsilon)$ when the grid is significantly under-resolved, i.e. at the coarse mesh resolution $\left(64^{3}\right)$. As the mesh resolution increases, any improvement offered becomes less apparent. An advantage of very high-order methods, such as the $9^{\text {th }}$ - and $11^{\text {th }}$-order WENO schemes, is the excellent scaling they achieve on high-performance computers (HPC) as demonstrated by Ri- 
tos, Kokkinakis, and Drikakis ${ }^{34}$. The computational overhead of the stencil normalization according to Eq. (7) is found to be small $(<1 \%)$ when applied to the computationally intensive $11^{\text {th }}$-order WENO scheme.

\section{COMPRESSION RAMP}

SBLI over a compression ramp inclined at $24^{\circ}$ angle was numerically investigated by $\mathrm{Wu}$ and Martin ${ }^{16}$ using DNS with free-stream conditions of Mach $=2.9$ and $R e_{\delta_{0}}=38,700$. The presence of the inclined surface gives rise to a shock wave, which interacts with the incoming turbulent boundary layer leading to the formation of a separation bubble and a $\lambda$-shock wave structure. The intensity of the incoming turbulent flow and the strength of the formed shock wave dictate the length of the separation region. A schematic illustrating the most important physical processes that take place is shown in Fig. 2.

\section{A. Boundary conditions}

Periodic boundary conditions are used in the spanwise $(y)$ direction while in the wall-normal $(z)$ direction a no-slip isothermal wall $\left(T_{w}=309 \mathrm{~K}\right)$ is used. We impose a supersonic outflow condition at the outlet and upper boundary opposite to the wall. The boundary condition at the inlet requires accurately assigning a turbulent boundary layer (TBL). The synthetic turbulent digital filter approach of Rana, Thornber, and Drikakis ${ }^{62}$, further modified in the present numerical study, is employed to generate the incoming turbulent boundary layer data.

The synthetic turbulent inflow boundary condition uses the digital filter (DF) method previously developed ${ }^{18,63,64}$ and validated $^{3,62,65}$ in the framework of the present DNS code CNS3D. Apart from the mean turbulent profile data, which can be obtained from either previous studies or flat plate simulations, the digital filter technique requires also knowledge of the integral length scales. In this study, an integral length scale of $L_{0 x}=0.22 \delta_{0}$ is used for the streamwise direction, whereas for the spanwise and wall-normal directions, the integral length scales are $L_{0 y}=0.2 \delta_{0}$ and $L_{0 z}=0.5 \delta_{0}$, respectively.

The digital filter technique used herein effectively creates a "synthetic" $2 \mathrm{D}$ random field of the state variables $\left(\mathbf{q}_{S}\right)$ to mimic that of a true turbulent field using known mean turbulent properties as input parameters. The approach is physically more representative of a TBL than simply using a white noise pseudo-random algorithm. The $2 \mathrm{D}$ perturbed flow properties are calculated similarly to Rana, Thornber, and Drikakis ${ }^{62}$, but only every $\Delta t_{S}=L_{0 x} / u_{\infty}$. At the intermediate time-instances the $\mathrm{BC}$ is set by interpolating two synthetic turbulent inflow planar distributions according to:

$$
\mathbf{q}_{0}^{t}=w_{S} \mathbf{q}_{S}^{t_{1}}+\left(1-w_{S}\right) \mathbf{q}_{S}^{t_{2}}
$$

where

$$
w_{S}=e^{-\pi r_{t}}-r_{t} e^{-\pi}, \quad r_{t}=\frac{t-t_{1}}{t_{2}-t_{1}}=\frac{t-t_{1}}{\Delta t_{S}}
$$

$t$ is the current time, $t_{1}$ is the time of the previous synthetic turbulent field, and $t_{2}$ is the synthetic turbulent field at the future time $t_{1}+\Delta t_{S}$, such that $t_{1} \leq t \leq t_{2}$. Alternatively, $\Delta t_{S}$ in Eq. (23) can also be estimated separately per mean boundary layer height location, i.e. according to the local mean velocity profile data rather that the free-stream value.

As the digital filter results in a perturbation of the velocity field only, appropriate fluctuations for the pressure and density need to be estimated. Invoking the Strong Reynolds Analogy (SRA) means that the pressure fluctuations in the boundary layer are considered negligible, i.e., $p^{\prime}=0$. Thus, the following relations are used to obtain the density and temperature fluctuations:

$$
\frac{\rho^{\prime}}{\bar{\rho}}=-\frac{T^{\prime}}{\bar{T}}
$$

and

$$
\frac{T^{\prime}(y, z)}{\bar{T}(z)}=-(\gamma-1) \bar{M}^{2}(z)\left[1+\frac{c_{p}\left(\bar{T}_{w}-\bar{T}_{t e}\right)}{\bar{u}(z) \bar{u}_{e}}\right] \frac{u^{\prime}(y, z)}{\bar{u}(z)}
$$

where $T_{w}$ is the wall temperature, $\bar{M}(z)=\bar{u}(z) / \sqrt{\gamma R_{s} \bar{T}(z)}$ is the Mach number at height $z, u_{e}$ is the velocity at the boundary layer edge $(z \approx 0.99 \delta), u^{\prime}(z, y)$ is the instantaneous planar velocity fluctuation field obtained by the digital filter technique, and

$$
\bar{T}_{t e}=T_{e}\left(1+\frac{\gamma-1}{2} M_{e}^{2}\right)
$$

is the total temperature at the boundary layer edge. Equation (25) was first introduced by Cebeci and Smith ${ }^{66}$ in the instantaneous form in an attempt to account for wall heat transfer effects. Gaviglio ${ }^{67}$ then referred to the root-mean-square (RMS) form of this relation as an extended strong Reynolds analogy (ESRA).

\section{B. Flow properties}

An incoming supersonic turbulent boundary layer (TBL) with a freestream Mach number of $M_{\infty}=2.9$ and a Reynolds number based on the boundary layer thickness of $R e_{\delta_{0}}=$ 38,737 is considered. The physical properties and computational domain are similar to previous DNS studies ${ }^{16}$ and further details are provided in Tables I and II, respectively.

Though the synthetic inflow perturbations cleverly "mimic" (resemble) that of a fully turbulent flow, the resulting incoming turbulent-like solution still requires to re-adjust and evolve to the physically correct condition as per the numerical scheme and grid resolution. Therefore, the position of the DF inflow is placed a further five boundary layer heights $\left(\sim 5 \delta_{0}\right)$ upstream relative to the DNS. The spanwise length is also increased to investigate possible large-scale structures that may develop in the post-shock region after re-attachment has occurred. Note that henceforth, $x / \delta_{0}=0$ will refer to the location of the compression corner. 


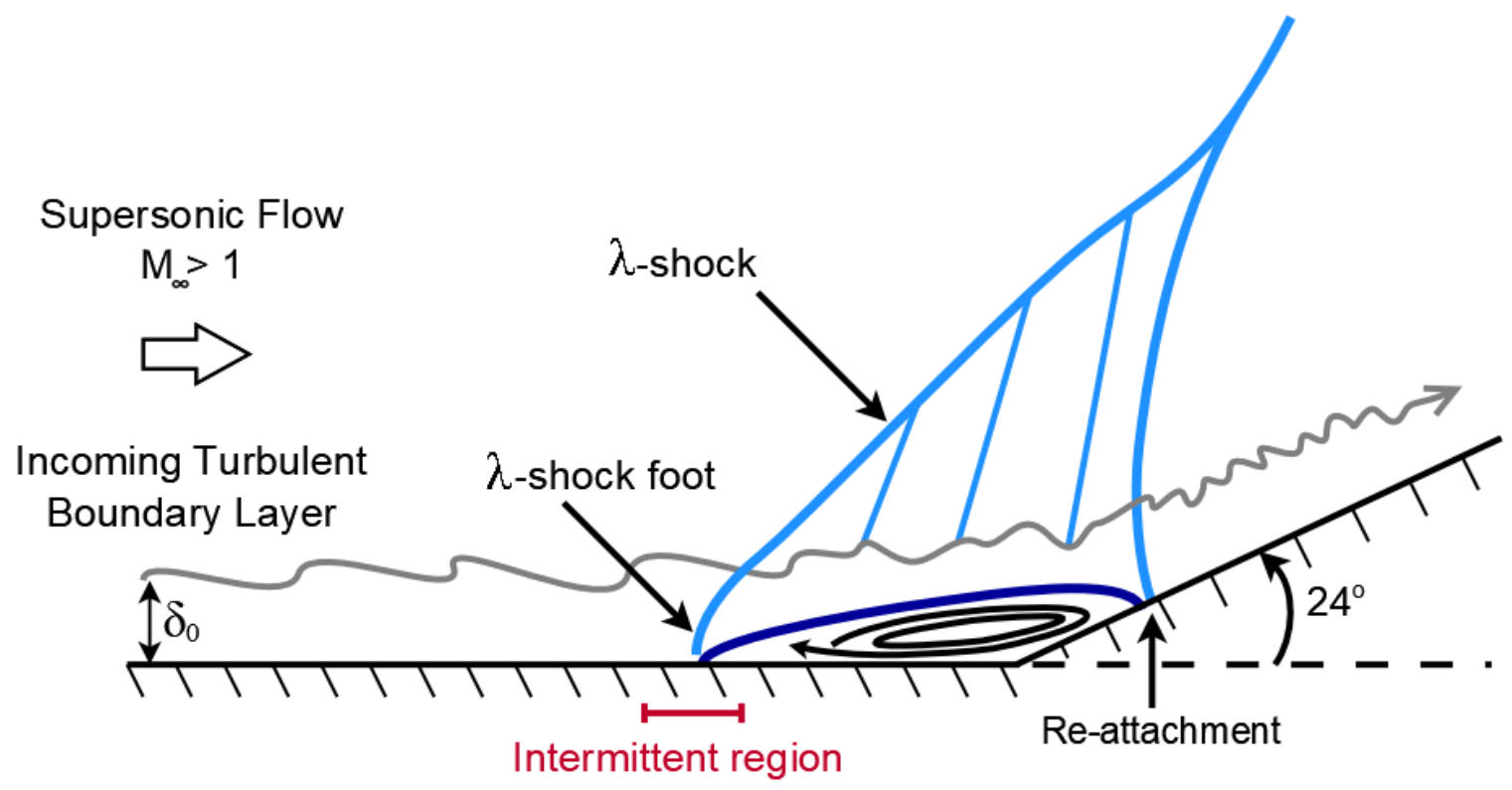

FIG. 2. Schematic illustrating the flow over a compression ramp.

TABLE I. Flow properties at the inflow for $M_{\infty}=2.9$

\begin{tabular}{lllll}
\hline \hline$\delta_{0}(\mathrm{~m})$ & $U_{\infty}(\mathrm{m} / \mathrm{s})$ & $T_{w}(\mathrm{~K})$ & $\rho_{\infty}\left(\mathrm{kg} / \mathrm{m}^{3}\right)$ & $T_{\infty}(\mathrm{K})$ \\
\hline 0.0064 & 609.1 & 307 & 0.077 & 107.1 \\
\hline \hline
\end{tabular}

\section{Grid parameters}

Prior knowledge of the wall unit can be obtained using empirical relations ${ }^{68}$. From the experiment of Bookey et al. ${ }^{8}$, both the values of the boundary layer momentum thickness, $\theta=0.43 \mathrm{~mm}$, and thickness, $\delta=6.7 \mathrm{~mm}$, are known. Using the relation proposed by Bies ${ }^{69}$ :

$$
\theta=\frac{\delta}{10.4+0.5 M^{2}\left(1+2 \times 10^{-8} \operatorname{Re}_{x}\right)^{1 / 3}}
$$

it is possible to obtain an estimate for $R e_{X}$, the Reynolds number based on the distance from the leading edge. A reasonable value of the friction coefficient can then be obtained using the Falkner formula:

$$
C_{f}=\frac{2 \tau_{w}}{\rho_{\infty} U_{\infty}^{2}} \approx 0.0263 \operatorname{Re}_{x}^{1 / 7}
$$

as proposed by Smol'yakov ${ }^{70}$. An estimate for the $y^{+}$value can thus be finally obtained according to $y^{+}=y u_{\tau} / v_{w}$, where the friction velocity is defined as $u_{\tau}=\sqrt{\tau_{w} / \rho_{w}}$. However, in order to calculate $y^{+}$, an estimate for $\rho_{w}$ and $v_{w}=\mu_{w} / \rho_{w}$ are also necessary. Assuming that the static pressure does not change significantly across the turbulent boundary layer, the density at the wall can be obtained according to the perfect gas law:

$$
\rho_{w}=\frac{p}{R_{s} T_{w}}
$$

where $R_{S}=c_{v}(\gamma-1)$ is the specific gas constant. The dynamic viscosity at the wall can be obtained by setting $T=T_{w}$ in Eq. (4).

Solving Eq. (26) for $R e_{x}$ and using the experimental values for $\delta$ and $\theta(6.4 \mathrm{~mm} \text { and } 0.43 \mathrm{~mm})^{8}$ returns $R e_{x} \approx 3.69 \times$ $10^{7}$, which once substituted into Eq. (27) gives a coefficient of friction of $C_{f} \approx 0.00218$. This empirically obtained value is very close -slightly lower- to the measured experimental value of 0.00225 and -slightly larger- to the DNS value of $0.00217^{16}$. According to Eq. (28), the density at the wall is approximately $\rho_{w} \approx 0.0285 \mathrm{~kg} / \mathrm{m}^{3}$, and the dynamic viscosity according to Sutherland's Law Eq. (4) is $\mu_{w} \approx 1.94 \times 10^{5}$. Thus, for $y^{+}=1$ a $\Delta y \approx 2.143 \times 10^{-5}$ meters is estimated, close to the value of $\Delta y \approx 2.16 \times 10^{-5} \mathrm{~m}$ used in the reference DNS $^{16}$.

The density and kinematic viscosity at the wall could also be obtained using the following two expressions, respectively:

$$
\rho_{w}=\rho_{\infty} \frac{T_{\infty}}{T_{w}}, \quad v_{w}=v_{\infty} \frac{\rho_{\infty}}{\rho_{W}}\left(\frac{T_{w}}{T_{\infty}}\right)^{r}
$$

while in the case of a adiabatic wall (zero heat-transfer), the wall temperature $\left(T_{w} \equiv T_{a w}\right)$ can be calculated by

$$
T_{a w}=T_{\infty}\left[1+\frac{r(\gamma-1)}{2} M^{2}\right]
$$

where $r=0.896$ is the recovery factor.

Following typical resolution recommendations for LES and DNS simulations ${ }^{71-73}$, a fine mesh for conducting wallresolved DNS is utilized. The mesh is clustered near the corner in the streamwise direction and near the wall in the wall-normal direction. The number of mesh points and corresponding mesh spacing are summarized in Table II along with the LES and DNS recommendations from the literature. The 


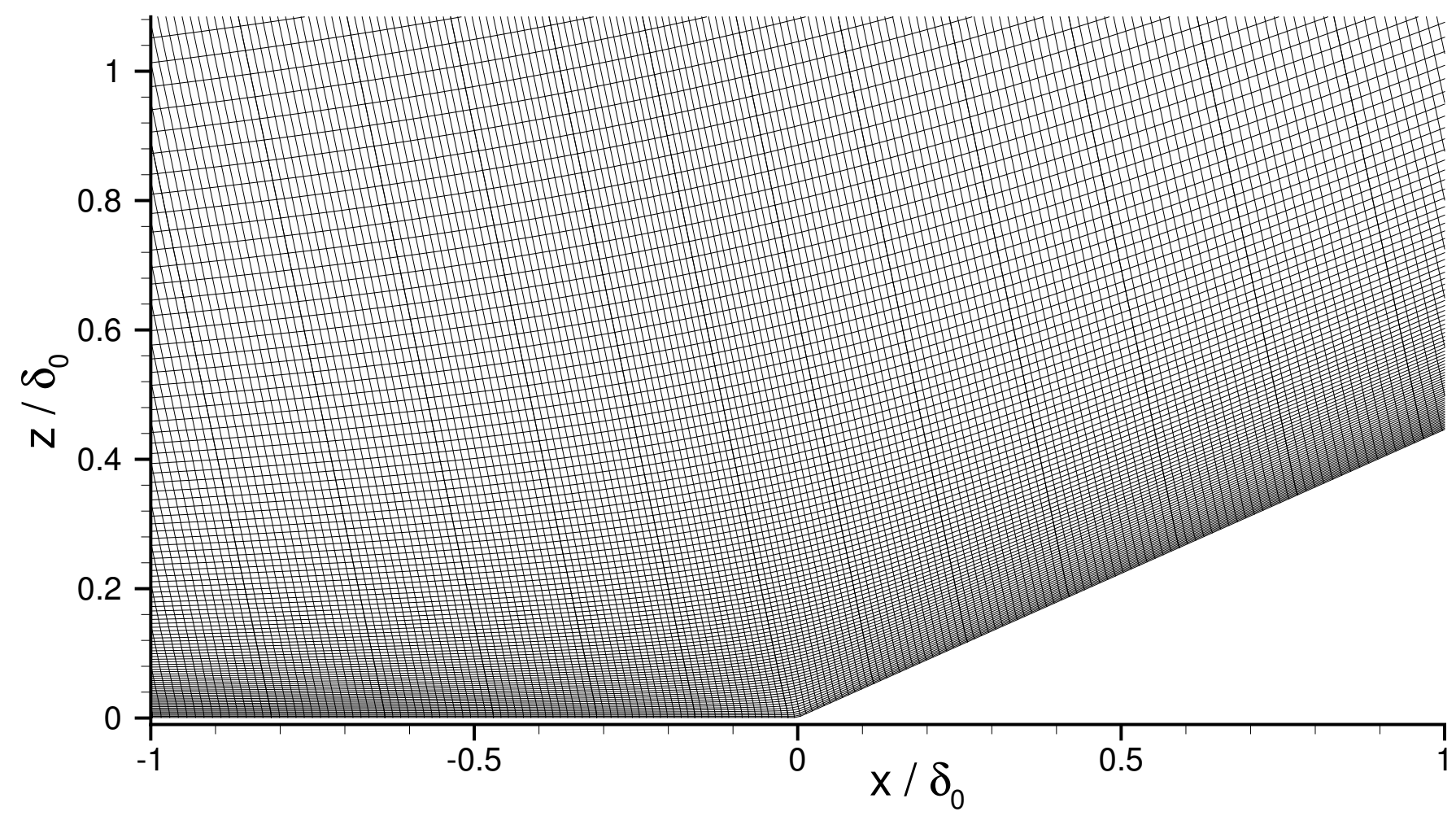

FIG. 3. Illustration of the structured curvilinear computational mesh employed; zoomed view at the location of the compression corner.

TABLE II. Mesh parameter comparison between the present DNS and the simulations of Wu and Martin ${ }^{16}$; number of cells given.

\begin{tabular}{llllllllll}
\hline \hline & $L_{x}$ & $L_{y}$ & $L_{z}$ & $n_{x}$ & $n_{y}$ & $n_{z}$ & $\Delta x^{+}$ & $\Delta y^{+}$ & $z^{+}$ \\
\hline DNS (present) & 21.4 & 3.0 & 5.0 & 1128 & 120 & 168 & 5.9 & 7.4 & 1.0 \\
DNS $^{16}$ & 15.4 & 2.2 & 5.0 & 1023 & 159 & 127 & 7.2 & 4.1 & 0.2 \\
\hline \hline
\end{tabular}

present mesh spacing $(\Delta y)$ is scaled using the conventional inner variable method $\Delta y^{+}=u_{\tau} \Delta y / v_{w}$, where $u_{\tau}=\sqrt{\tau_{w} / \rho_{w}}$ is the friction velocity, $v_{w}, \tau_{w}$ and $\rho_{w}$ are the near-wall kinematic viscosity, wall shear-stress and density, respectively. A previous mesh convergence study ${ }^{44}$ demonstrated that the current mesh resolution is sufficiently fine to achieve highly accurate results. Grid clustering is also used in the streamwise direction. At the compression ramp corner, $\Delta x^{+} \approx 2.93$; a value of 3.4 was quoted for the same location in the DNS ${ }^{16}$. Figure 3 shows the mesh around the compression corner within $\delta_{0}$ radius.

\section{RESULTS}

We initially performed the simulations for $\sim 200 \delta_{0} / u_{\infty}$ to allow the turbulent inflow condition to exit the outlet of the domain several times to achieve a statistically steady state. The simulations were carried out for a further $\sim 300 \delta_{0} / u_{\infty}$ to obtain a statistically steady average representation of the turbulent flowfield, with three-dimensional flowfield data collected at intervals of $\sim 2 \delta_{0} / u_{\infty}$.
Kokkinakis and Drikakis ${ }^{44}$ previously demonstrated that the LM correction of Thornber et al. ${ }^{36}$ particularly improved the accuracy of low-order schemes $\left(\leq 5^{\text {th }}\right)$ in subsonic turbulent boundary layer flows. Therefore, we will examine here the LM correction only in context with the $2^{\text {nd }}$ (M2LM) and $5^{\text {th }}$-order MUSCL schemes (M5LM).

We have examined the correlation properties of the turbulent inflow resulting from the digital filter technique by estimating the $2^{\text {nd }}$ order correlation $\left(r_{i j}\right)$ and its correlation coefficient $\left(R_{i j}\right.$ - the auto-covariance of the fluctuating velocity $\left.u_{i}^{\prime}\right)$ :

$$
\begin{aligned}
r_{i j}(\mathbf{x}, \eta, \tau) & =\overline{u_{i}^{\prime}(\mathbf{x}, t) u_{j}^{\prime}(\mathbf{x}+\eta, t+\tau)} \\
R_{i j}(\mathbf{x}, \eta, \tau) & =\frac{r_{i j}(\mathbf{x}, \eta, \tau)}{\sqrt{\overline{u_{i}^{\prime 2}}}(\mathbf{x}) \sqrt{\overline{u_{j}^{\prime 2}}}(\mathbf{x}+\eta)}
\end{aligned}
$$

The overbar denotes Reynolds time-averaging, where $\eta$ is the spatial and $\tau$ is the temporal separation. Here, we have considered the case $\tau=0$ and the $x$-direction. Figure 4 shows the three normal correlations $(i=j)$ at the digital filter inlet and at a distance of $0.1 \delta_{0}$ from the wall. All three correlations quickly drop to zero within about $0.5 \delta_{0}$ and after that begin 


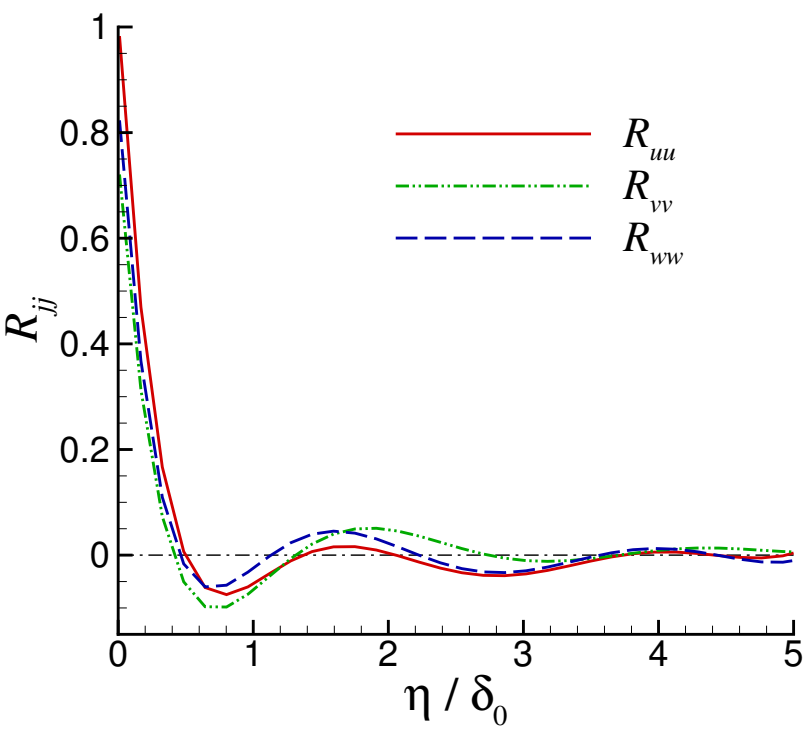

FIG. 4. Autocorrelation of velocity fluctuations in the streamwise direction at $z=0.1 \delta_{0}$ in the incoming boundary layer for the DNS.

to oscillate with a decaying amplitude as the downstream distance increases. The results suggest that a distance of $5 \delta_{0}$ is sufficient for the artificial turbulent inflow to decorrelate, thus not affecting the TBL statistics.

\section{A. Mean flow}

The streamwise distribution of the time and spatially averaged mean wall pressure (Fig. 5) shows that the W9 scheme provides more accurate results than the MUSCL schemes. The W9 results are in excellent agreement with both the DNS of Wu and Martin ${ }^{16}$ and experiment of Bookey et al. ${ }^{8}$. M5LM (green line) does not correctly capture the location of the separation bubble because the resolution of finer flow structures is weaker compared to W9. The above numerical behavior leads to the formation of more energetic large scale turbulent structures, which are responsible for "blowing" the shock in the downstream direction. Our experience is that W9 can attain the same accuracy with the M5LM while using a coarser mesh ${ }^{33-35,68,74}$.

The friction coefficient, $C_{f}$, is calculated according to the first equality in Eq. (27) and is plotted in Fig. 6. The lowMach number correction (LMC) does not improve $C_{f}$ significantly due to the subsonic region of the undisturbed TBL confined within the viscous sublayer. Though the subsonic region at the separation bubble can extend as high as $0.6 \delta_{0}$, also evident by the sonic line depicted in Fig. 10, the position of the shock remains largely unaffected. Any loss of accuracy in this critical region should lead to significant errors in the SBLI dynamics, as well as the turbulent flow characteristics downstream. However, analysis of the turbulence kinetic energy budget terms carried out later in $\S \mathrm{VD}$ and shown in Fig. 15, reveals that the turbulence is relatively weak below the sonic line. Therefore, the LMC is found to offer only a marginal

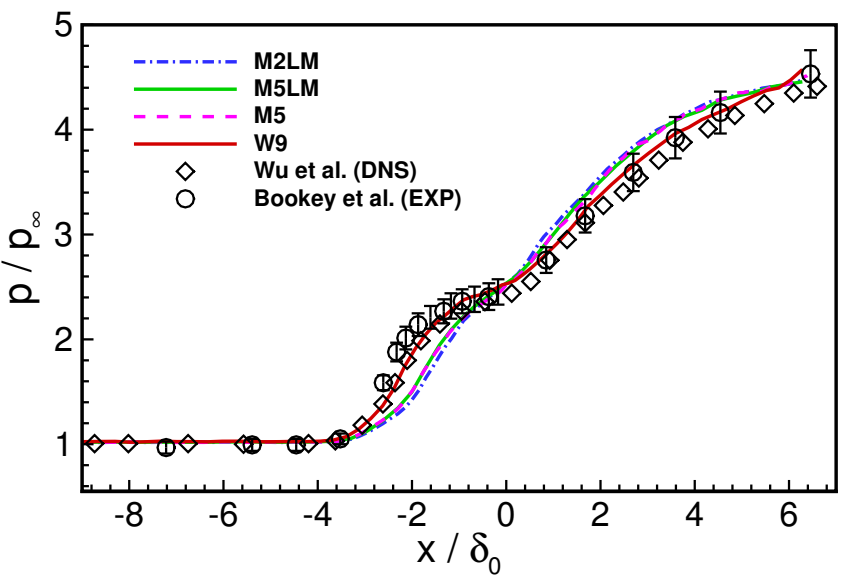

FIG. 5. Normalized mean wall pressure, $p / p_{\infty}$, distribution along the streamwise direction.

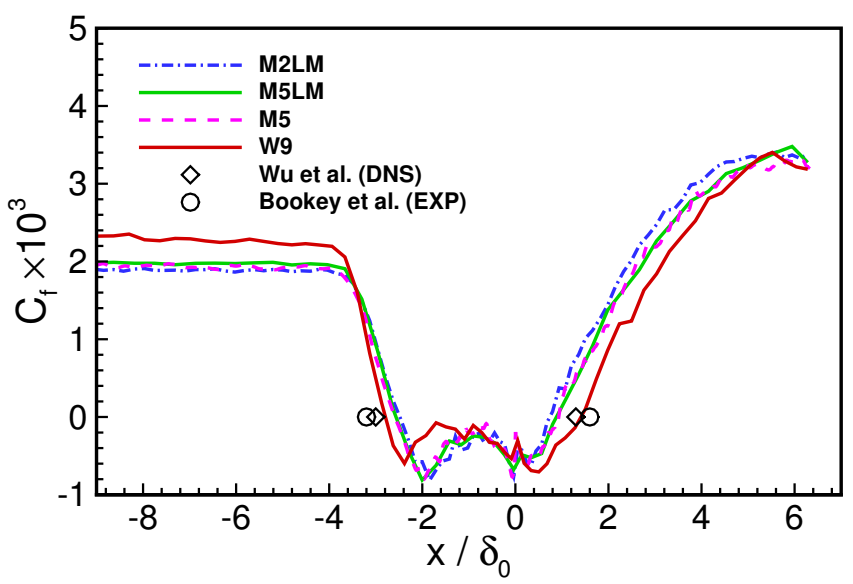

FIG. 6. Skin friction coefficient, $C_{f}$, distribution along the streamwise direction.

improvement of accuracy in this case.

The spatial order of the scheme has a substantial effect on simulation accuracy. At $x=-8 \delta_{0}$, the W9 accurately predicts the skin friction coefficient, $C_{f} \approx 0.0022$, whereas both the M5 and M2LM underestimate it: $C_{f} \approx 0.00195$ and 0.0019 , respectively. The LMC improves the skin friction coefficient of the fifth-order MUSCL scheme (M5LM) by $2.5 \%$ $\left(C_{f} \approx 0.002\right)$. The corresponding value quoted by the $\mathrm{DNS}^{16}$ and experimental ${ }^{8}$ studies was $C_{f} \approx 0.00217$ and 0.00225 , respectively. The W9 gives the closest agreement to the experimental locations at which the $C_{f}$ changes sign, which indicates the extent of the SBLI separation bubble. The results suggest that the ability of the scheme to accurately resolve the turbulent structures in the supersonic region of the "undisturbed" TBL largely determine the accuracy in the prediction of the size and location of the separation bubble.

Nonetheless, the computational overhead of the LMC is found to be small, as also observed in previous studies ${ }^{44,45}$. The increasing accuracy of the W9 compensates for the higher computational cost. As shown in ${ }^{2,44}$, low-order schemes require much finer grids, and smaller time-step sizes, ultimately 
turning into an increased computational cost.

A comparison of the mean streamwise velocity profile at $-11 \delta_{0}$ and $-8 \delta_{0}$ reveals the streamwise evolution of the TBL created by the digital filter technique. The position $x=-8 \delta_{0}$ corresponds to the inflow boundary condition of the reference $\mathrm{DNS}^{16}$, which used a re-scaling method developed by $\mathrm{Xu}$ and Martin ${ }^{75}$ to generate the inflow condition, with the recycling station located $4.5 \delta_{0}$ downstream of the inlet. Thus, to make a credible comparison of the present DNS data to the reference DNS, it is paramount that - at the very least - the mean flow properties, such as the mean velocity profile, agree well at the same location upstream of the compression ramp corner $\left(x=-8 \delta_{0}\right)$.

The mean streamwise velocity profile at $x=-8 \delta_{0}$ (Fig. 7) shows excellent agreement to the DNS and experiment for all three schemes investigated. However, the W9 is more accurate near the wall $\left(z<0.1 \delta_{0}\right)$, but only marginally in the remainder of the profile. The synthetic turbulent field created by the digital filter technique required approximately $6 \delta_{0}$ in the streamwise direction to adjust, with the results suggesting a minimum distance of around $3-4 \delta_{0}$ for the current synthetic turbulence inflow implementation.

Once the flow reaches the intermittent region - where the $\lambda$-shock foot interacts with the separation bubble formed as illustrated in Fig. 2 - there is a considerable difference between the three schemes in the mean streamwise velocity profile (Fig. 7(b)). The W9 gives the best agreement to the reference DNS profile at this location, a consequence of the more precise size and location of the resolved separation bubble. The differences between the second- (M2LM) and fifth-order (M5LM) MUSCL schemes are much less significant, though the M5LM shows some slight improvement.

Figure 7 (c) plots the velocity tangential to the wall at $x=$ $6.1 \delta_{0}$ near the outlet boundary:

$$
\tilde{u}_{\|}=\tilde{u} \cos \left(24^{\circ}\right)+\tilde{v} \sin \left(24^{\circ}\right)
$$

versus normal to the wall along the line defined by:

$$
\frac{z}{\delta_{0}}=\tan \left(24^{\circ}-90^{\circ}\right)\left(\frac{x}{\delta_{0}}-6.1\right)+6.1 \tan \left(24^{\circ}\right)
$$

All three mean velocity profiles show some deviation from the reference DNS but at different sections of the boundary layer profile. The W9 scheme closely matches the DNS profile in the upper layer $\left(z \approx 2.3 \delta_{0}\right)$, which is indicative of having resolved the position of the oblique shock wave and, in turn, the position of the separation bubble leading edge. Both the M2LM and M5LM are in closer agreement to the reference DNS in the inner re-attached TBL region $z<0.5 \delta_{0}$; however, we believe that this is incidental to the stencil in this region and further investigation is required.

A qualitative comparison of the schemes accuracy can be accomplished by plotting the isosurfaces of the coherent turbulent structures (vortices). We have used the compressible $Q_{M}$-criterion $^{76,77}$, defined as:

$$
Q_{M}=Q+I I_{S}>0
$$

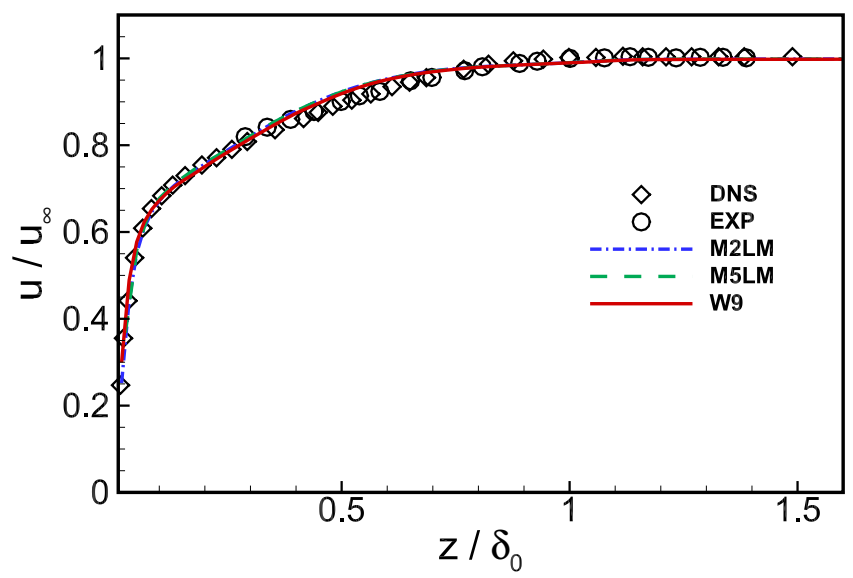

(a) $x=-8.0 \delta_{0}$

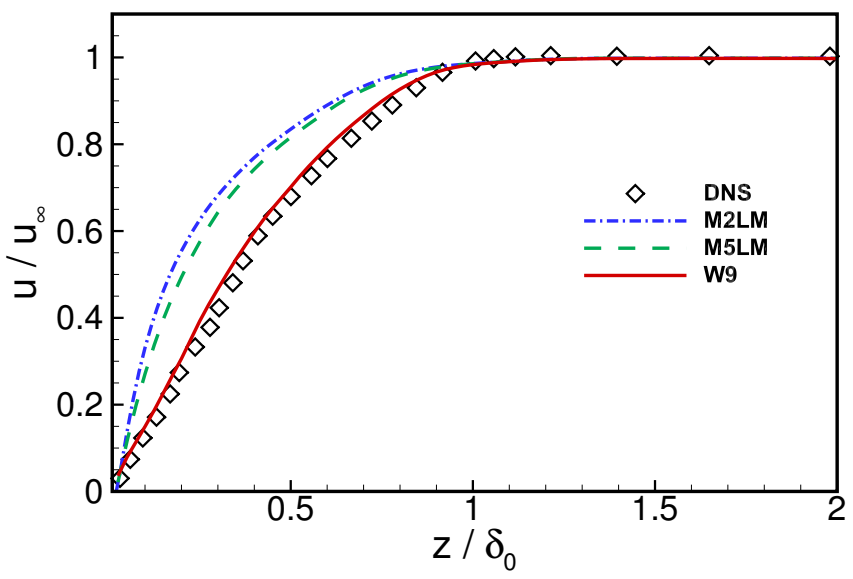

(b) $x=-1.9 \delta_{0}$

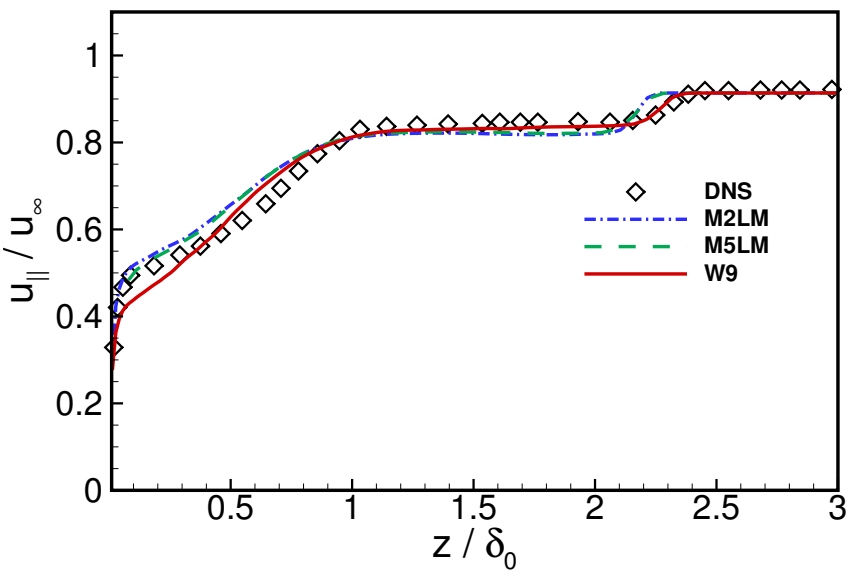

(c) $x=6.1 \delta_{0}$

FIG. 7. Comparison of inflow streamwise velocity to the reference DNS $^{16}$ and Experiment ${ }^{8}$ at three different locations: (a) $x=-8.0 \delta_{0}$, (b) $x=-1.9 \delta_{0}$, and (c) $x=6.1 \delta_{0}$.

where the standard (incompressible) $Q$-criterion is given by:

$$
Q=\frac{1}{2}\left(\|\Omega\|^{2}-\|\mathrm{S}\|^{2}\right)
$$


and $I I_{S}$ is the second invariant of the strain-rate tensor $S$

$$
I I_{S}=\frac{1}{2}\left[\operatorname{Tr}(\mathrm{S})^{2}-\operatorname{Tr}\left(\mathrm{S}^{2}\right)\right]
$$

$\nabla \mathbf{u}$ consists of the symmetric strain-rate tensor $S$ and antisymmetric vorticity tensor $\Omega$ :

$$
\mathrm{S}=\frac{1}{2}\left[\nabla \mathbf{u}+(\nabla \mathbf{u})^{T}\right], \quad \Omega=\frac{1}{2}\left[\nabla \mathbf{u}-(\nabla \mathbf{u})^{T}\right]
$$

and the standard Frobenius tensor norm for a tensor $\mathrm{T}$ in three dimensions takes the form

$$
\|\mathrm{T}\|=\sqrt{\operatorname{Tr}\left(\mathrm{TT}^{T}\right)}
$$

The $Q_{M}$ isosurfaces (Fig. 8) reveals that increasing the spatial order of the scheme provides a greater abundance of flow structures, particularly upstream of and around the SBLI. In the relaxation region downstream of the SBLI, the differences become less apparent. The W9 resolves much finer scale vortices throughout the height of the TBL. As a result, the W9 transfers and "injects" more effectively into the viscous sublayer a larger amount of mean kinetic energy, leading to an increase in the resolved wall shear-stress (Fig. 6). The mechanism is that W9 captures the details of the vortices at the turbulent/nonturbulent interfaces (TNTI), which carry turbulent fluctuations into the TBL. This process takes place simultaneously with the fluctuations in the freestream, which are radiated from inside the TBLs as reported by Duan, Choudhari, and Zhang ${ }^{78}$.

Although the freestream flow is supersonic, the boundary layer in the lower portion of TBL remains subsonic, comprising approximately 20 computational cells off the wall for the grid employed in this study. According to Figure 9, the "undisturbed" (pre-SBLI) TBL is subsonic up to $z^{+} \approx 11$, which contains the viscous sublayer and the lower part of the buffer layer. The data indicates that only the streamwise Reynolds stress $\left(\mathrm{R}_{x x}\right)$ peaks within the subsonic TBL region; $\mathrm{R}_{i j}$ is defined later in Eq. (38). Both the maximum spanwise $\left(\mathrm{R}_{y y}\right)$ and wall-normal $\left(\mathrm{R}_{z z}\right)$ Reynolds stress components occur in the supersonic region of the TBL, with the former located as high as $z^{+} \approx 45$, around the beginning of the loglaw layer. The turbulent motion transferred from higher-up the TBL towards the no-slip wall is primarily governed by the wall-normal fluctuations located in the supersonic portion of the TBL. In terms of magnitude, the streamwise fluctuations are much stronger than the wall-normal components, $\mathrm{R}_{x x} \approx 5.4 \mathrm{R}_{z z}$, although nearer the wall viscous forces become increasingly dominant.

\section{B. Analysis of low-frequency oscillation}

The mixing layer is identified as the region of high-density gradient magnitude located between the separation bubble and primary oblique shock wave (Fig. 10); that is the portion of the TBL that detaches from the plain surface and later re-attaches at the inclined surface (ramp). The secondary compression

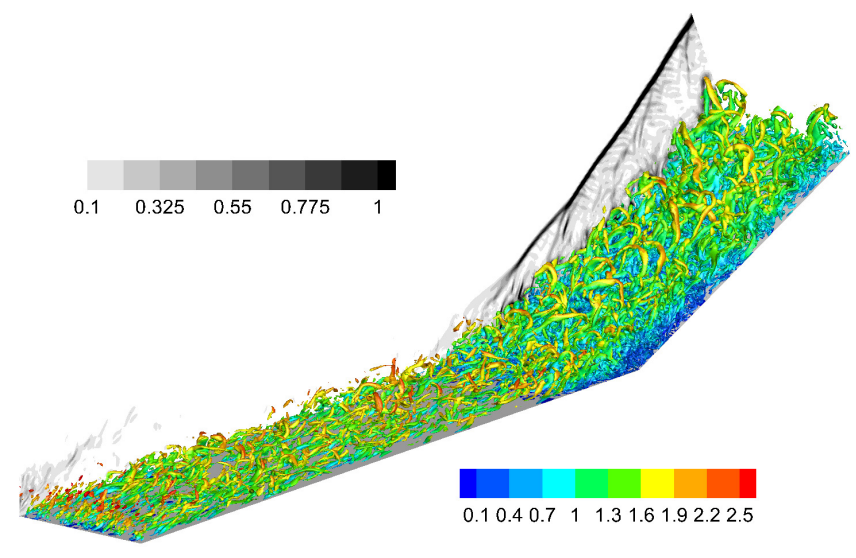

(a)

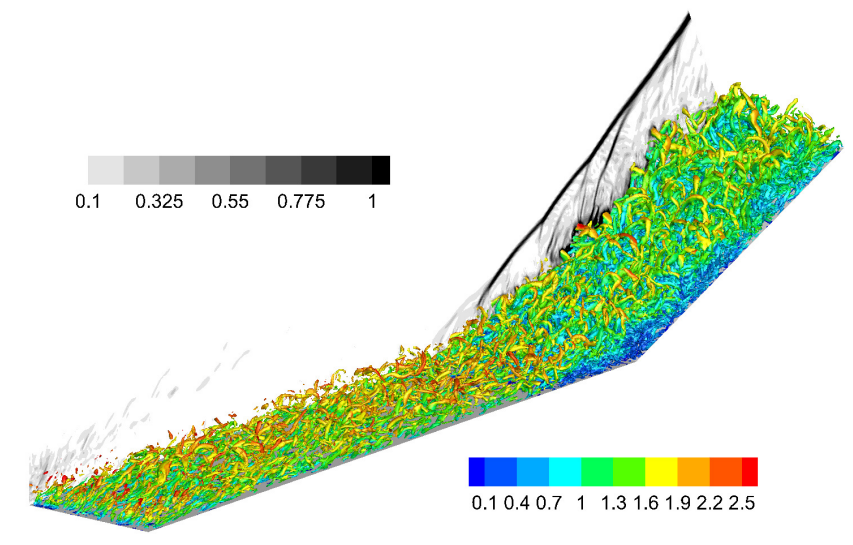

(b)

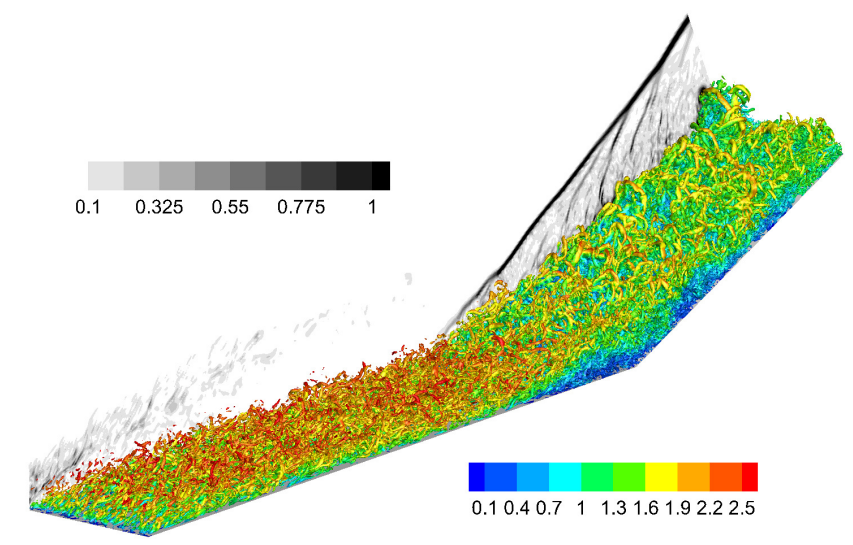

(c)

FIG. 8. Isosurfaces of $Q_{M}$-criterion $\left(2 U_{\infty}^{2} / \delta_{0}^{2}\right)$ coloured by Mach number and $2 \mathrm{D}(x-z)$ contour plane of density gradient magnitude $|\nabla \rho| \delta_{0} / \rho_{\infty}$ in grayscale using schemes (a) M2LM, (b) M5LM, and (c) W9.

waves, or shocklets, are visible above the mixing layer along the mean pressure isobar $\bar{p} / p_{\infty}=3$, and merge onto the primary shock; these are also visible, to some extent, at the contour surface (grayscale density gradient), Fig. 8. The above isobar terminates onto the ramp surface very close to the reattachment of the mixing layer. Moreover, a change in the 


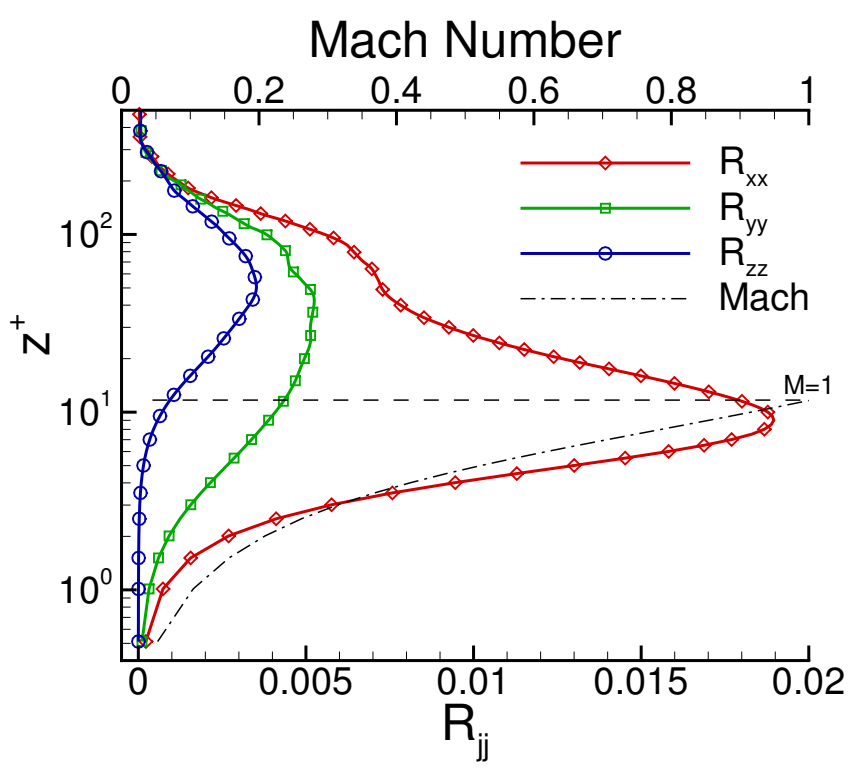

FIG. 9. Normalized normal Reynolds stress components, $\mathrm{R}_{j j} / u_{\infty}^{2}$, at $x=-9 \delta_{0}$.

angle of the mixing layer occurs as it is intersected by the $\bar{p} / p_{\infty}=3$ isobar, effectively splitting it into two distinct regions. The diffuse appearance of the mean secondary compression waves in Fig. 10 is indicative of their large scale oscillatory behavior, i.e. the wide spatial range over which they form.

We have performed power spectral density (PSD) analysis of the surface pressure loading only for the W9 scheme, as it provides the best agreement with the experimental measurements ${ }^{8}$. The position of the numerical pressure probes is shown in Fig. 11: one prior to SBLI at $x / \delta_{0}=-6.9$ (Point 1), and two before and after the separation bubble onset at $x / \delta_{0}=-2.98$ (Point 2) and -2.18 (Point 3), respectively. The separation bubble onset is taken as the point at which the friction coefficient, $C_{f}$, changes sign. For the W9 scheme, it occurs approximately at $x / \delta_{0} \approx-2.785$.

The PSD at the three locations is plotted in Fig. 12. Both pressure and frequency have been non-dimensionalized using outer-layer scaling; the freestream pressure normalizes the fluctuating pressure signal, $p^{\prime}(t) / p_{\infty}$, while the frequency, $f$, is normalized according to $S t=f \delta_{0} / u_{\infty}$, where $S t$ is the dimensionless Strouhal number. We show the frequency up to $S t \sim 10$, which is near the grid resolution cut-off at the wall, i.e. $f_{\max } \sim s_{w} /\left(2 \Delta y_{w}\right)$, where $s_{w}=\sqrt{\gamma R_{s} T_{w}}$ is the speed of sound at the wall and $\Delta y_{w}$ is the largest cell edge; in this case in the spanwise (y) direction.

For the undisturbed supersonic TBL, the peak PSD is at the non-dimensional frequency of one. The energy is slowly transferred from the large scales to $f \approx 1$ (henceforth $f \equiv S t$ ). In general, the PSD follows the established cascade ${ }^{79}$ that has been investigated for compressible flows ${ }^{35}$, and defines four distinct frequency domains:

1. Low frequency $f^{x}$ : it is influenced by the turbulent motion in the outer part of the boundary layer, while the viscosity and turbulent motion influence high frequencies in the inner part of the boundary layer. For incompressible flows $x=2$, while for compressible flows $x \rightarrow 0$.

2. Mid frequency: it includes the spectral peak $\left(f^{0}\right)$;

3. Mid-to-High overlap frequency $f^{-r}$ where $r=[0.7-$ 1.1]: it appears at sufficiently high $R e$ values and $r$ is influenced by the local Reynolds number. This region is associated with pressure-induced eddies in the logarithmic region of the boundary layer.

4. High frequency $f^{-s}$ : The spectrum scales with $s=7 / 3$ in a region called "acoustic-transition". At higher frequencies the spectrum decays more rapidly $(s=5)$ due to the viscous sublayer $\left(z^{+}<20\right)$ and weaker compressibility (local mean Mach number $<1$ ).

Figure 12 reveals that the high-frequency domain scales as $f^{-5}$ in all three locations. The same behavior was also observed by Bernardini, Pirozzoli, and Grasso ${ }^{80}$. Inhibition of the higher frequencies is not observed downstream of the separation zone due to the sustained high-speed flow after the oblique shock. This effect is in contrast to the case of a normal shock, also associated with subsonic flow pocket as previously reported ${ }^{80}$.

The mid-to-high frequency band (undisturbed TBL) is found to be very narrow since the Reynolds number is not sufficiently high for it to become perceptible. At the third point located within the separation region, a much broader mid-tohigh overlap region forms, which is attributed to the breakdown and redistribution of the large-scale (or low-frequency mode) energy content previously formed at the front of the separation zone (point 2). Although the mean streamwise velocity at position 2 is positive, its instantaneous variation contains negative values. The above suggests that the separation bubble leading-edge oscillates in the streamwise direction over time.

The abrupt increase in the PSD content at the low-end frequency range of the wall pressure signal at $x=-2.98 \delta_{0}$ (Fig. 12 - red line), alludes to the presence of a large scale structure. This is in agreement with the observations made by Bernardini, Pirozzoli, and Grasso ${ }^{80}$ and further supported by the similarity of the near-wall pressure fluctuation PSD in the mid- to high-end frequency band between the $\lambda$ shock foot $\left(x=-2.98 \delta_{0}\right)$ and the undisturbed supersonic TBL $\left(x=-6.9 \delta_{0}\right)$.

The speed of sound at the wall (point 2) and the low-end frequency range $f \in[0.04,0.08] u_{\infty} / \delta_{0}$ over which the PSD abruptly decreases, suggest a flow structure of a time-scale of $[12.5,25] \delta_{0} / u_{\infty}$. We have ruled out unsteadiness of the recirculation vortex within the separation zone as the source of the sudden frequency change at point 2 . This is because the time required for a full convective recirculation (of the outer streamline) corresponds to the significantly lower frequency of $\sim 0.01 u_{\infty} / \delta_{0}$.

Given the above, we believe that an acoustic (pressure wave) mechanism is present. The acoustic propagation time required to travel along streamline $\Psi$ from the separation 


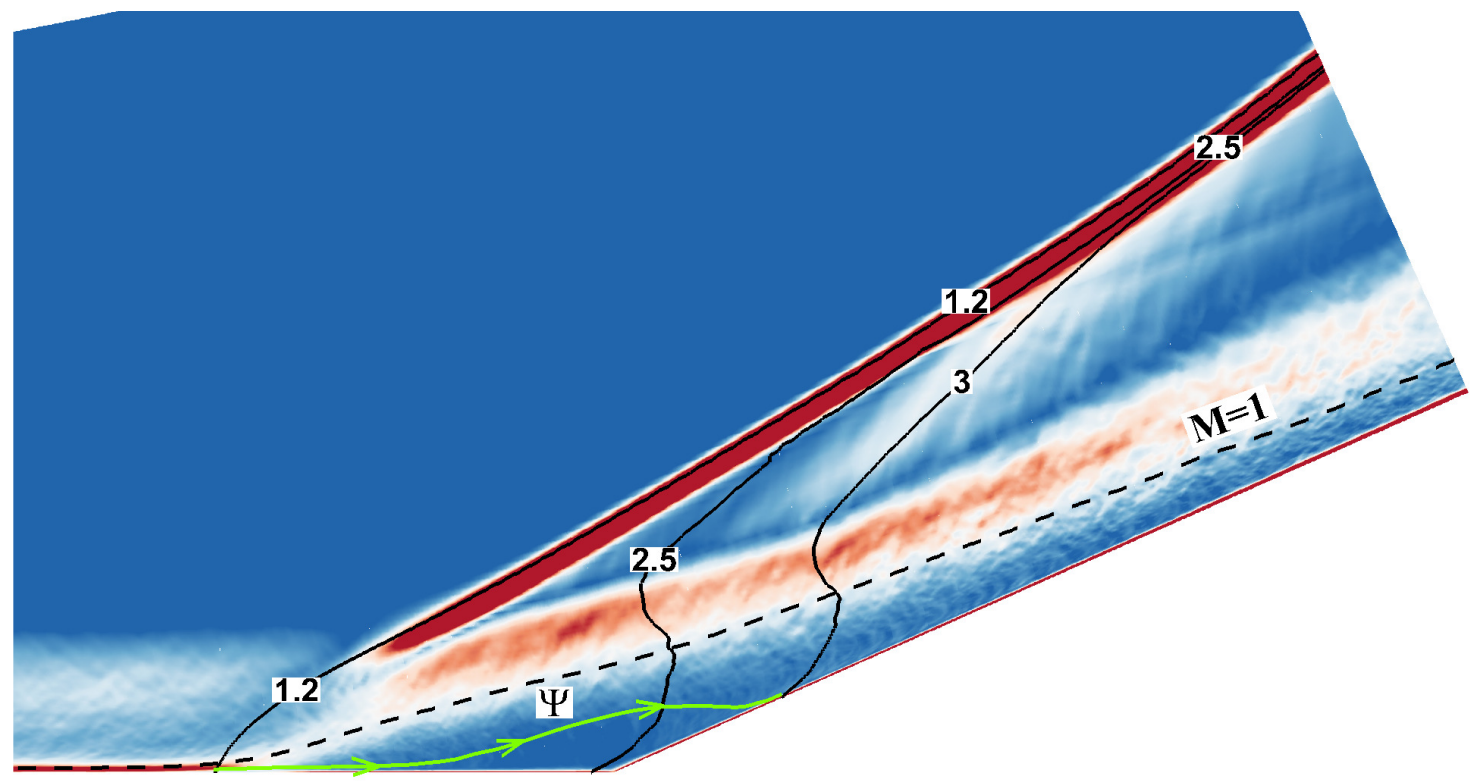

FIG. 10. Contour plot of mean density gradient magnitude $\left(\nabla \bar{\rho} \delta_{0} / \rho_{\infty}\right)$; solid lines of $\bar{p} / p_{\infty}$, dashed sonic line $(M=1)$, green line with arrows depicts streamline $\Psi$ over the recirculation bubble

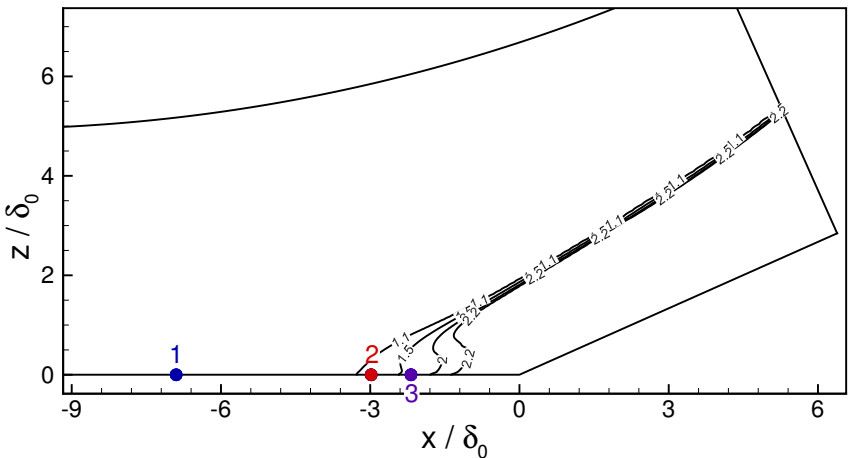

FIG. 11. Illustration of the location of the numerical probes relative to the SBLI; contour-lines of $p / p_{\infty}$.

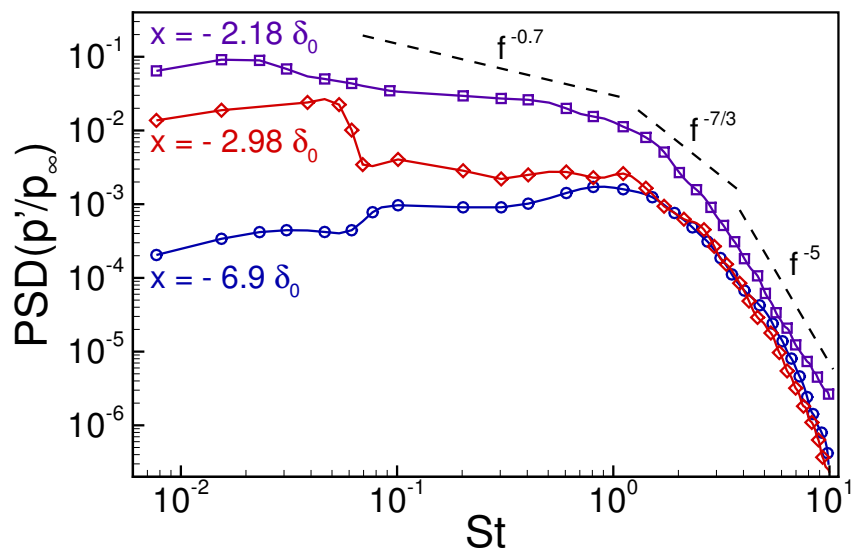

FIG. 12. Power spectral density (PSD) of pressure signal at the three probe locations; Welch function. point to the re-attachment position in the relaxation region, can be estimated by carrying out a line integral along the streamline (path) $\Psi$ joining $(x, z) / \delta_{0}$ from $\sim(-2.98,0.028)$ to $\sim(1.368,0.623)$ :

$$
\Delta t_{\Psi}=\int_{\Psi}(\gamma p / \rho)^{-1 / 2} \mathrm{~d} \psi
$$

where $\mathrm{d} \psi$ is the length of a discrete segment along streamline $\Psi$, i.e. $\mathrm{d} \psi=\sqrt{\mathrm{d} x^{2}+\mathrm{d} z^{2}}$. The streamline $\Psi$ is depicted in Fig. 10 by the green solid line with arrows.

Equation (37) gives a time-scale of $\Delta t_{\Psi} \approx 8 \delta_{0} / u_{\infty}$. Note that the relatively large region of subsonic Mach number postSBLI allows acoustic waves to propagate upstream of the reattachment towards the SBLI. The mean convective transport is ignored since its effect on the acoustic wave propagation cancels out when carrying out both forward and backward integration along the streamline path.

Assuming that the acoustic wave travels along streamline $\Psi$ from the point of the mean flow separation to mean reattachment and back, it gives us an estimated time-scale of $\sim 16 \delta_{0} / u_{\infty}$. The above is within the observed low-frequency range $[12.5,25] u_{\infty} / \delta_{0}$ found to correspond to the abrupt increase in the pressure fluctuation PSD at the $\lambda$-shock foot in Fig. 12. We can obtain a value closer to the upper range value of $25 u_{\infty} / \delta_{0}$ by integrating the acoustic speed propagation upstream along the wall surface. Nonetheless, $\Delta t_{\Psi}$ in itself does not constitute sufficient evidence which unequivocally proves that the observed low-frequency mechanism at the $\lambda$-shock foot is induced by pressure reciprocity between the SBLI separation and re-attachment regions. The exact physical mechanism leading to this phenomenon is not yet well understood, and further analysis is required. 
We hypothesize that unsteadiness in the secondary compression waves, which are formed at the re-attachment of the mixing layer in the relaxation region, causes a sufficiently significant increase in the local mean pressure. The pressure increase influences the upstream SBLI via the (substantial) subsonic region present post-SBLI within the separation zone and mixing layer, as evident by the mean sonic line in Fig. 10. The local mean compression is attributed to the abrupt impingement (re-attachment) of the turbulent mixing layer onto the ramp surface.

Schneider ${ }^{81}$ showed that weak harmonic waves could propagate upstream in a supersonic stream along a plane wall over distances that are very large in comparison with the boundarylayer thickness. However, the upstream propagation distance depends on the width of the subsonic layer. As the Reynolds number increases, the sonic line rapidly approaches the wall. In the experiment of Settles, Fitzpatrick, and Bogdonoff $^{12}$, the SBLI set-up was similar to the present study. The Reynolds number was of an order of magnitude higher. In the experiment, the sonic line in the undisturbed TBL was around $0.01 \delta$ from the wall, i.e. $0.04 \delta_{0}$ of the present case. However, the shock was still prone to unsteady low-frequency oscillations. Though previous studies have shown that the extent of the upstream influence is an active function of the Reynolds number ${ }^{82,83}$, and it becomes weaker with increasing Reynolds number, a separation layer and a subsonic region will nonetheless still develop. The subsonic region can be several orders of magnitude more extensive in the post-shock area as Fig. 10 illustrates. The acoustic interaction, or reciprocity, mechanism proposed here should be further investigated for different ramp angles, higher Reynolds and Mach cases.

\section{Reynolds stress anisotropy}

We have investigated numerical accuracy regarding the turbulent anisotropy using the barycentric map method of Banerjee et $a l .{ }^{84}$. To enhance the clarity between the different turbulence anisotropy states, we have instead implemented a modified form of the map first suggested by Emory and Iaccarino ${ }^{85}$. A description of the procedure follows.

The Reynolds stress, $\mathrm{R}_{i j}$, and the Reynolds stress anisotropy tensor, $\mathrm{a}_{i j}$, are calculated according to:

$$
\mathrm{R}_{i j}=\widetilde{u_{i}^{\prime \prime} u_{j}^{\prime \prime}}=\overline{\rho u_{i}^{\prime \prime} u_{j}^{\prime \prime}} / \bar{\rho}
$$

and

$$
\mathrm{a}_{i j}=\frac{\mathrm{R}_{i j}}{2 k}-\frac{\delta_{i j}}{3}, \quad \text { where } k=\frac{\mathrm{R}_{k k}}{2}
$$

The three eigenvalues of the $a_{i j}$ (diagonal) tensor are obtained such that $\lambda_{1} \geq \lambda_{2} \geq \lambda_{3}$.

We use the eigenvalues to position the turbulence field on the barycentric anisotropy invariant map ${ }^{84}$. We show that the eigenvalues lie in the interval $-1 / 3 \leq \lambda_{i} \leq 2 / 3$ and that the number of non-zero eigenvalues and equalities between them can identify the limiting states of anisotropy. The limiting states are known as spherical, pancake-like and rod or cigarlike turbulence based on their visual description.
The first state corresponds to isotropic turbulence, or otherwise a three-component state, i.e. $X_{3 c}\left(\lambda_{1}=\lambda_{2}=\lambda_{3}=0\right)$. The above state represents turbulence where fluctuations exist in all directions at equal magnitude. The next state is the isotropic two-component turbulence $\mathrm{X}_{2 c}\left(\lambda_{1}=\lambda_{2}=1 / 6, \lambda_{3}=\right.$ $-1 / 3)$, which describes turbulence where fluctuations exist along two directions with equal magnitude. The last limiting state is one-component turbulence $\mathrm{X}_{1 c}\left(\lambda_{1}=2 / 3, \lambda_{2}=\lambda_{3}=\right.$ $-1 / 3)$, which describes a flow where turbulent fluctuations only exists along one direction.

A convex combination of the above limiting states can describe any turbulence state. An equilateral triangle is constructed to visualise the barycentric map. The peaks of the graph represent the limiting states (Fig. 13). We can identify both the intensity and the type of turbulence anisotropy. A modified weights estimate ${ }^{85}$ is used to further distinguish the different transition regions between the three limiting states of anisotropy:

$$
\mathrm{X}_{i c}=\left(C_{i c}+C_{o f f}\right)^{C_{e x p}}
$$

where

$$
\begin{aligned}
& C_{1 c}=\lambda_{1}-\lambda_{2} \\
& C_{2 c}=2\left(\lambda_{2}-\lambda_{3}\right) \\
& C_{3 c}=3 \lambda_{3}+1
\end{aligned}
$$

and $C_{\text {off }}=0.65$ and $C_{\text {exp }}=5$.

The sides of the triangle (map boundaries) define the transition regions. The right side of the triangle, coloured magenta in Fig. 13, corresponds to axisymmetric expansion in which one diagonal component of the Reynolds stress tensor is larger than the other two, equal components. Thus turbulence has a prolate spheroid shape. The left side of the triangle, coloured cyan, represents an axisymmetric contraction in which one component is smaller than the other two parts, which are equal. Therefore, turbulence has an oblate spheroid shape. The bottom side of the triangle, coloured yellow, shows the two-component turbulence which can be visualized as an ellipse and is typically present near the solid walls where the wall-normal component of the fluctuations vanishes much faster than the other parts.

The tiny artificial perturbations generated at the digital filter inflow saturate the freestream Reynolds stress anisotropy componentality. Therefore, each normal Reynolds stress component is filtered when the velocity magnitude becomes greater or approximately equal to the freestream, i.e. if $|\mathbf{u}| \lesssim$ $0.997 u_{\infty}$ then $\mathrm{R}_{i i}=4 e^{-5} u_{\infty}^{2}$.

The results from the three numerical schemes, as evidenced by Fig. 13, produce anisotropy invariant maps with minimal differences between each other. A similar conclusion was also previously drawn in the case of a supersonic plane turbulent boundary layer flow ${ }^{33}$. A higher-order scheme just leads to a sharper boundary defining the different componentality (colour) regions, causing the latter to be identified more readily. The freestream flow is purely isotropic and covers the majority of the domain away from the boundary layer. The shock 

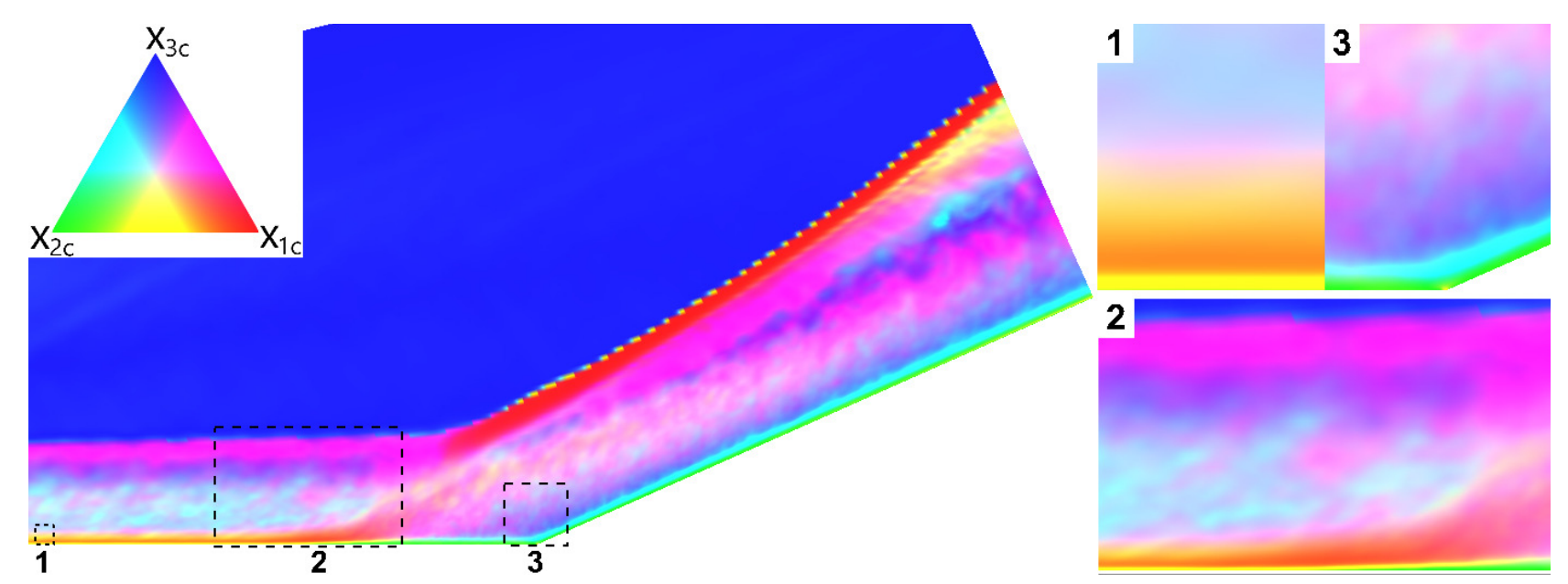

(a)
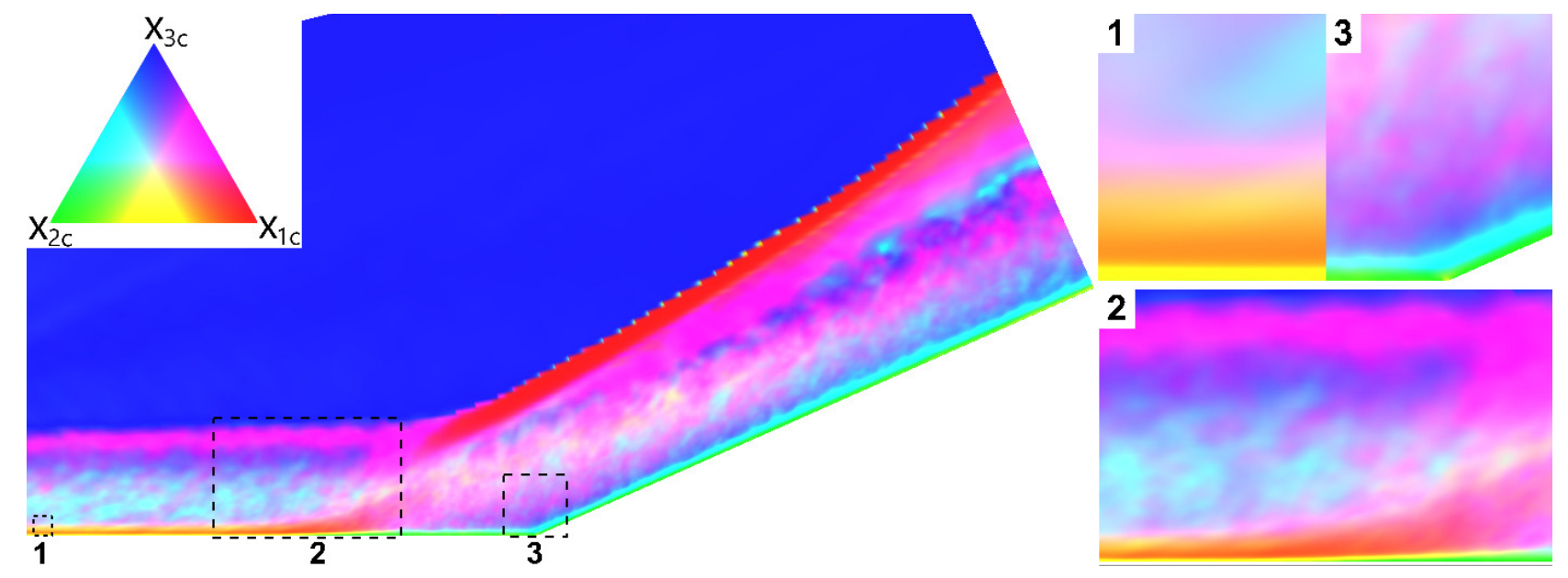

(b)
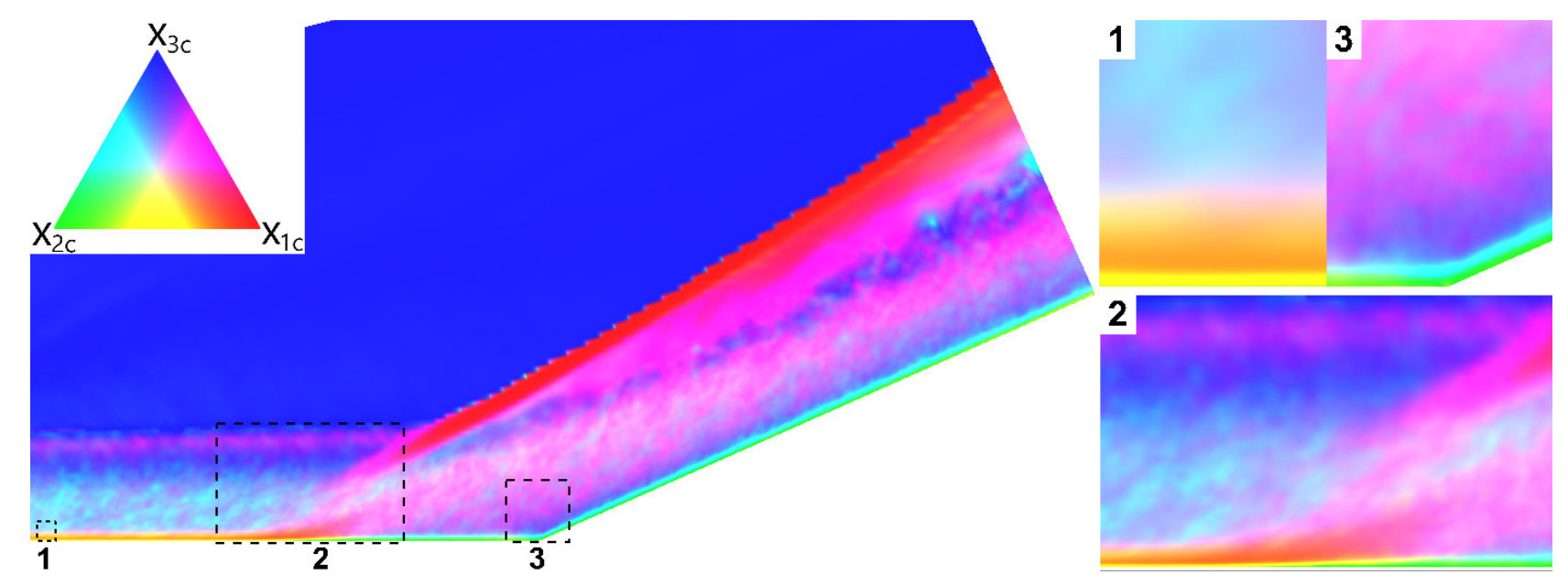

(c)

FIG. 13. Contour plots of Reynolds stress anisotropy componentality for (a) M2LM, (b) M5LM and (c) W9 using Eq. (39) with $C_{o f f}=0.65$ and $C_{\text {exp }}=5$. 


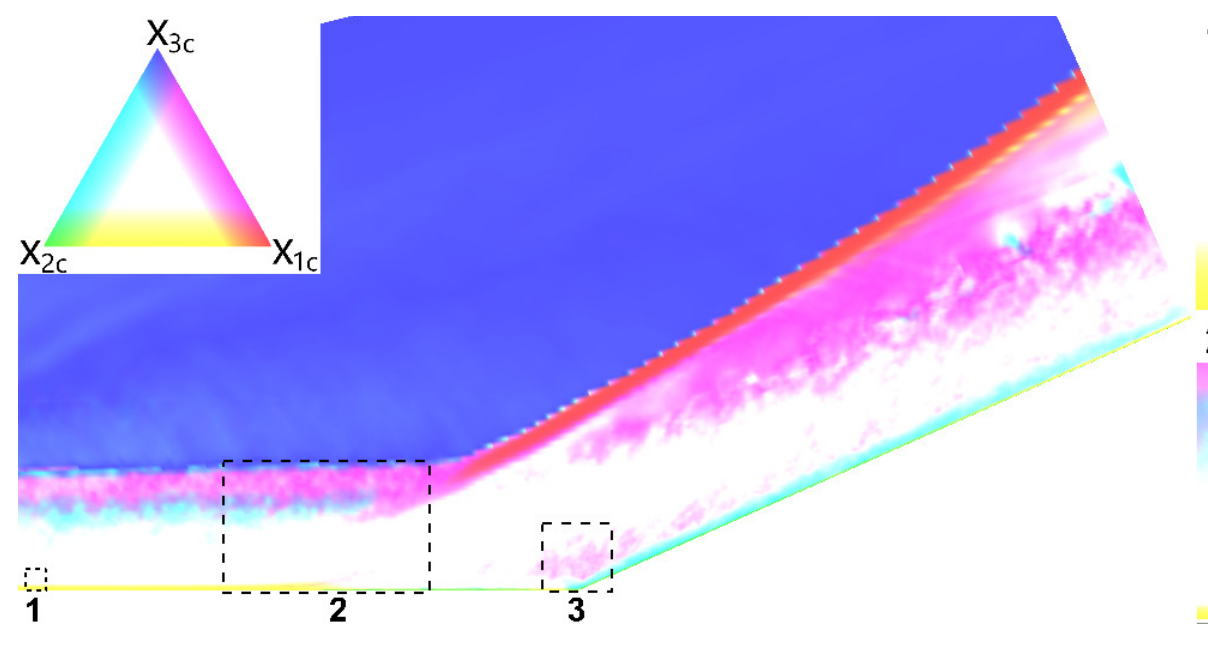

FIG. 14. Contour plots of Reynolds stress anisotropy componentality for W9 using Eq. (39) with $C_{o f f}=0.80$ and $C_{\exp }=5$.

itself is highlighted by a layer of one component turbulence as expected.

In the undisturbed TBL and adjacent to the wall, we observe (as expected) a thin layer of two-component turbulence (yellow colour). Slightly above the wall, a layer of onecomponent turbulence exists (red colour). Both regions are commonly encountered in the viscous sublayer and buffer layer of a TBL, respectively; the former is indicative of the elliptic disc-like structures within the viscous sublayer, while the latter of the long streaky structures that develop just above it in the buffer layer. Outside of the buffer layer, a significant portion of the log-law region comprises turbulent structures alternating between a state of axisymmetric contraction (oblate) and expansion (prolate). Though sufficient averaging should result in a single colour, it is shown later, in Fig. 14, that this is due to small alternations off the centre of the current colour-map. Nonetheless, this still eludes to the presence of a mix of oblate and prolate turbulent structures that dominate the log-law region of the undisturbed TBL.

In the outer region of the TBL, close to the edge, a distinctive colour alteration from blue to magenta and back to blue occurs. We believe that the isotropic limit below the TBL edge is a result of the high degree of mixing between the oblate and prolate turbulent structures present below. The magenta colour at the TBL edge indicates the dominating presence of prolate spheroid-like structures (axisymmetric expansion), which are the result of turbulent horseshoe vortices (Fig. 8). Above the undisturbed TBL, the rod-like structures escape into the freestream and quickly dissipate to three-component isotropic turbulence. The authors had made a similar observation in the past ${ }^{33}$ while tracking the path of the Reynolds stress componentality along with the height of a supersonic plane turbulent boundary layer on a barycentric invariant map.

Downstream of the shock and in the proximity of the ramp wall, the two-component limit turbulence (yellow) transforms to two-component axisymmetric limit (green). In contrast, the one-component turbulence layer (red) turns to axisymmetric contraction (cyan) turbulence. Outwards, the turbulence transforms to three-dimensional turbulent structures (blue) followed by a sizeable axisymmetric expansion (magenta) region. The latter part before the SBLI was predominantly composed of turbulence under axisymmetric contraction and the change is attributed to the effect of the shock wave (amplification of the streamwise Reynolds stress).

Using the anisotropy colour mapping Eq. (39) with constants $C_{o f f}=0.65$ and $C_{\text {exp }}=5$ leads to uncertainty around the centre point of the colour-map legend (equilateral triangle). Small variations off-centre but in different directions can result in significant colour alterations. The above causes the cyan and magenta colour alternation observed across most of the undisturbed supersonic TBL before the SBLI (Fig. 13). Instead, setting $C_{\text {off }}=0.8$ in Eq. (39) returns an alternative colour-mapping that effectively "filters-out" a large portion of the colour map surrounding the centre of the barycentric map. The result is a colour map which essentially represents only the anisotropy states near the boundaries.

The alternative colour-map helps reveal several distinct features that were previously not discernible. The common thought is that post-re-attachment, in the recovery region, turbulence begins to evolve towards an equilibrium state again, i.e. develops a TBL anisotropy state similar to pre-SBLI. However, Fig. 14 suggests otherwise. A distinct magenta region forms in the outer region of the mixing layer, just above the compression ramp corner post-SBLI. Immediately, it begins to spread from the outer region of the re-attached mixing layer inwards and towards the wall, while occupying an evergreater proportion of the redeveloping TBL. The expansion of the anisotropy state is caused by the much larger magnitude of the streamwise Reynolds stress, $\left(R_{x x}\right)$, relative to the two normal stress components.

The turbulence in the vicinity of the primary shock is predominantly of a single component, as defined by the intense red colour in Figs. 13 and 14. We attribute the above behaviour to a significant increase in the wall-normal Reynolds stress component $\left(R_{z z}\right)$ relatively to the other two normal components (though $\mathrm{R}_{x x}$ increases too). The magenta region following the primary shock is the result of the shocklets unsteadiness, as well as of the amplification of the weak 
freestream turbulence radiated outward from the undisturbed supersonic TBL by the shock wave. Though the primary shock amplifies the wall-normal Reynolds stress component, it rapidly decays soon after. Consequently, the streamwise Reynolds stress becomes the dominant component in the magenta region found post-SBLI. In contrast, the magnitude of the normal Reynolds stress component is relatively much weaker $\left(\mathrm{R}_{i i}<5 \times 10^{-4} u_{\infty}^{-2}\right)$.

\section{Turbulence kinetic energy budget}

For a compressible flow the turbulence kinetic energy (TKE), $\tilde{k}=\operatorname{Tr}\left(\mathrm{R}_{i j}\right) / 2$, is given by

$$
\frac{\partial \bar{\rho} \tilde{k}}{\partial t}=\mathrm{C}+\mathrm{P}+\mathrm{T}_{d}+\mathrm{T}_{p}+\mathrm{D}_{v}-\varepsilon+\mathrm{M}+\Pi_{p}
$$

where the terms on the R.H.S. of Eq. (40) are given in Table III.

Similar to the Reynolds stress invariant maps, the numerical schemes predict very similar TKE budgets; thus, we present only the results for the $9^{\text {th }}$-order WENO (W9). Figure 15 shows the two-dimensional distribution of all the turbulence kinetic energy budget terms. The location of the SBLI significantly influences the turbulence kinetic energy (TKE). Relative to the SBLI location and post-SBLI flowfield, the magnitude of most budget terms is considerably lower in the undisturbed (upstream) TBL.

In most of the undisturbed TBL, the TKE transport term (C) tends to zero, representing the balance between the production and dissipation term, a typical characteristic of equilibrium turbulence (Fig. 15(a)). In the SBLI region, and more specifically in the $\lambda$-shock foot, the advective term sharply increases above the area of the peak of the production term (P). Thus, indicating the advective transfer of TKE towards the outer region of the free-shear layer. This process continues along the entire length of the free-shear layer up until the recovery region.

Following the re-attachment of the turbulent free-shear flow on to the ramp wall, the advective term attains large negative values in the lower half of the TBL, following a region of significant dissipation. In the outer edges of the re-attached TBL, the TKE due to advection begins to "mix" with the negative TKE region. Provided that the ramp is sufficiently long, we expect that these two regions would eventually cancel out as the TBL gradually regains an equilibrium state.

The peak value of the production term $\mathrm{P}$ (Fig. 15(b)) occurs (i) just above the separation bubble front in the vicinity of the shockwave and separated shear layer, and (ii) in a short region downstream of the separation point. The above behaviour is due to the strong mean shear sustained around these regions ${ }^{19}$.

In the undisturbed TBL, the production term $\mathrm{P}$ encompasses a peak around $y^{+} \approx 8-9$, that decays rapidly towards the viscous sublayer $\left(y^{+}<5\right)$ and more gradually along the log-law region, eventually disappearing at the wall and the TBL edge. The production term notably peaks in the buffer layer region $5<y^{+}<45$ and is thus the underlying turbulence producing region sustaining the supersonic TBL.
TABLE III. Turbulence kinetic energy budget terms.

\begin{tabular}{|c|c|}
\hline $\mathrm{C}=-\frac{\partial}{\partial x_{j}}\left(\bar{\rho} \tilde{k} \tilde{u}_{j}\right)$ & Advection \\
\hline $\mathrm{P}=-\overline{\rho u_{i}^{\prime \prime} u_{j}^{\prime \prime}} \frac{\partial \tilde{u}_{i}}{\partial x_{j}}$ & Production \\
\hline $\mathrm{T}_{d}=-\frac{\partial}{\partial x_{j}}\left(\overline{\rho u_{i}^{\prime \prime} u_{i}^{\prime \prime} u_{j}^{\prime \prime}}\right)$ & Turbulent diffusion \\
\hline $\mathrm{T}_{p}=-\frac{\partial}{\partial x_{j}}\left(\overline{p^{\prime} u_{j}^{\prime \prime}}\right)$ & Pressure diffusion \\
\hline $\mathrm{D}_{v}=\frac{\partial}{\partial x_{j}}\left(\overline{\tau_{i j}^{\prime} u_{i}^{\prime \prime}}\right)$ & Viscous diffusion \\
\hline$\varepsilon=\tau_{i j}^{\prime} \frac{\partial u_{i}^{\prime \prime}}{\partial x_{j}}$ & Dissipation \\
\hline $\mathbf{M}=\overline{u_{i}^{\prime \prime}}\left(\frac{\partial \bar{\tau}_{i j}}{\partial x_{j}}-\frac{\partial \bar{p}}{\partial x_{i}}\right)$ & Mass flux \\
\hline$\Pi_{p}=\overline{p^{\prime} \frac{\partial u_{i}^{\prime \prime}}{\partial x_{i}}}$ & Pressure strain / Dilatation \\
\hline
\end{tabular}

Away from the wall, the turbulence producing mechanism transitions from boundary layer turbulence to free-shear turbulence. In the recovery region post-SBLI, the production term decreases as a result of the decay of the free-shear layer. At the same time, the near-wall peak rises as a result of the flow re-attaching and reverting to a turbulent boundary layer.

The turbulent transport term $\mathrm{T}_{d}$ is most significant in the SBLI region and free-shear layer (Fig. 15(c)). In the undisturbed TBL (before SBLI), $\mathrm{T}_{d}$ increases from zero at the wall to a maximum positive value at $y^{+} \approx 4.5$ near the boundary between the viscous sublayer and buffer region. In contrast, in the range $6.4<y^{+}<19$ within the buffer layer, $\mathrm{T}_{d}$ is negative. This result is indicative of a diffusion process, which transfers TKE from regions where it is the largest (source) and reversely leading to an influx of TKE into lower-value areas (sink). The mechanism is identical to that reported for a canonical flat-plate TBL by Li et al. ${ }^{19}$.

In the near-wall region both pre- and post-SBLI, the turbulent transport/diffusion $\mathrm{T}_{d}$ and viscous diffusion $\mathrm{D}_{v}$ terms balance similarly. In the undisturbed TBL, the production of TKE peaks at $y^{+} \approx 9$, where the turbulent transport term $\mathrm{T}_{d}$ attains its most considerable negative value. The maximum positive value of $\mathrm{T}_{d}$ is within the upper viscous sublayer at $y^{+} \approx 4.3$. This result suggests that $\mathrm{T}_{d}$ transports TKE from the production region towards the outer viscous sublayer. On the other hand, the viscous diffusion term $\mathrm{D}_{v}$ attains its most substantial negative value at $y^{+} \approx 6.5$, while its positive value peaks at $y^{+} \approx 0.87$, similarly to the dissipation term $\varepsilon$. The above suggests that viscous diffusion is more effective at transporting TKE from the upper viscous sublayer and towards the wall. Apart from the more considerable value of $\mathrm{D}_{v}$ at $y^{+} \approx 0.87$, both terms attain a similar magnitude higher up the undisturbed TBL, suggesting that they work in tandem, transporting TKE from the central production region in the buffer layer, towards the near-wall area where it is dissipated.

Above the viscous sublayer of the undisturbed TBL, when 

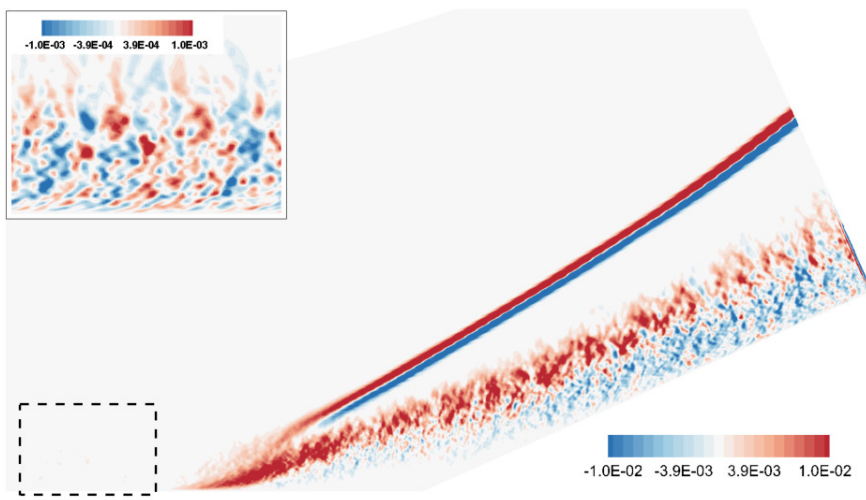

(a) $\mathrm{C}$

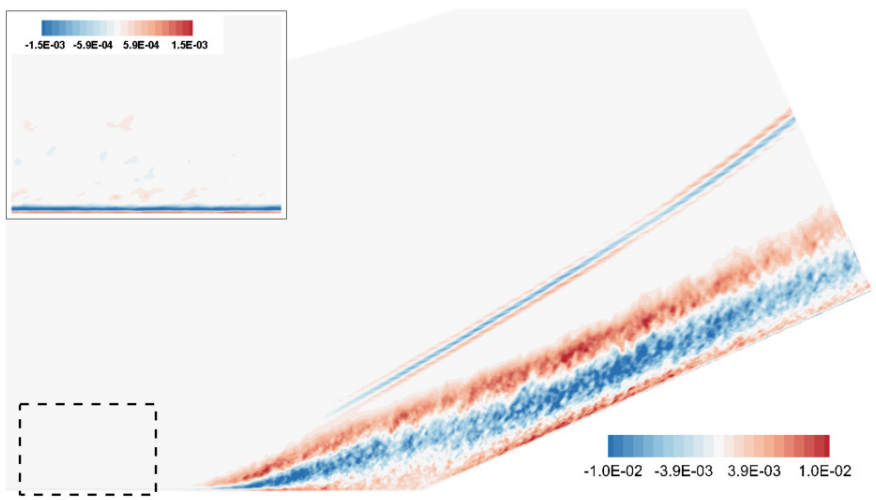

(c) $T_{d}$
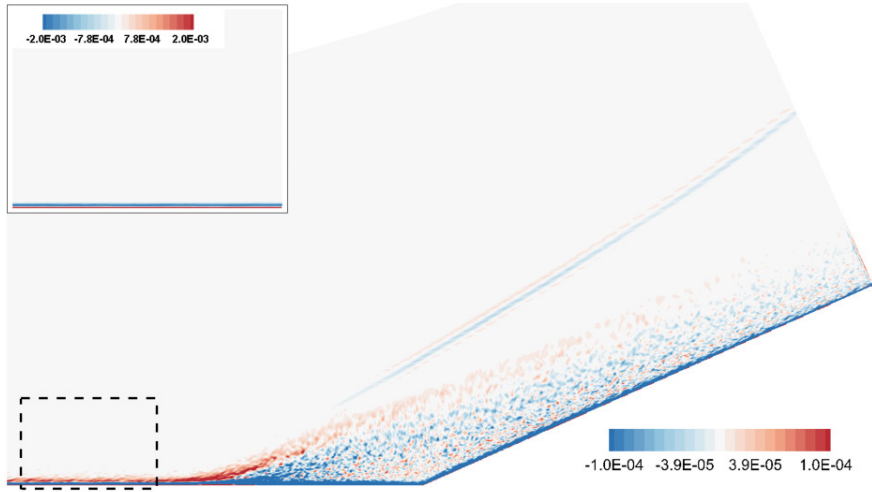

(e) $\mathrm{D}_{v}$
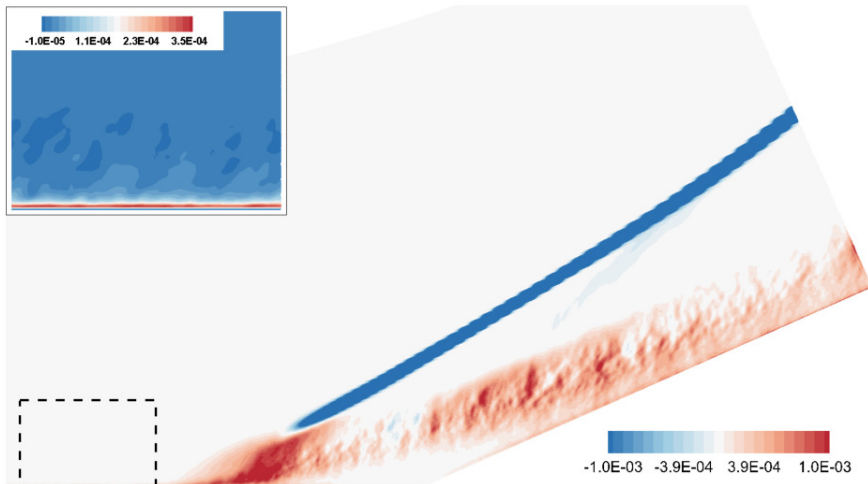

(g) $\mathrm{M}$

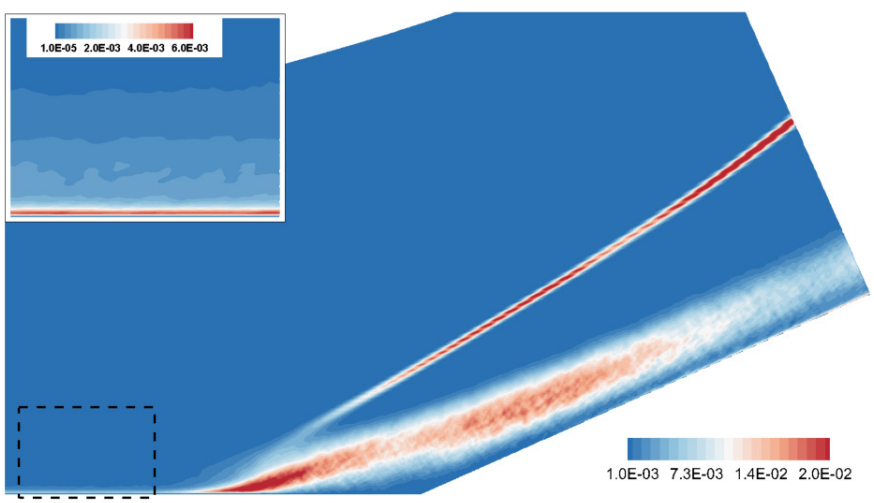

(b) $\mathrm{P}$

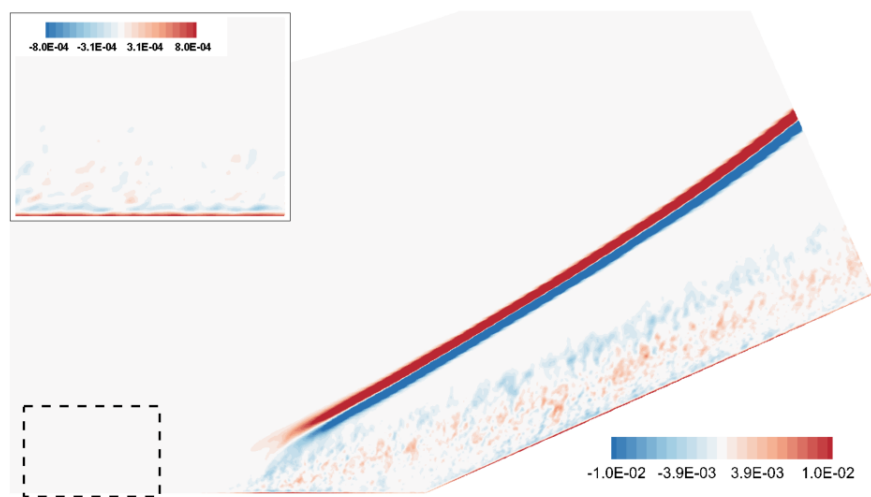

(d) $\mathrm{T}_{p}$

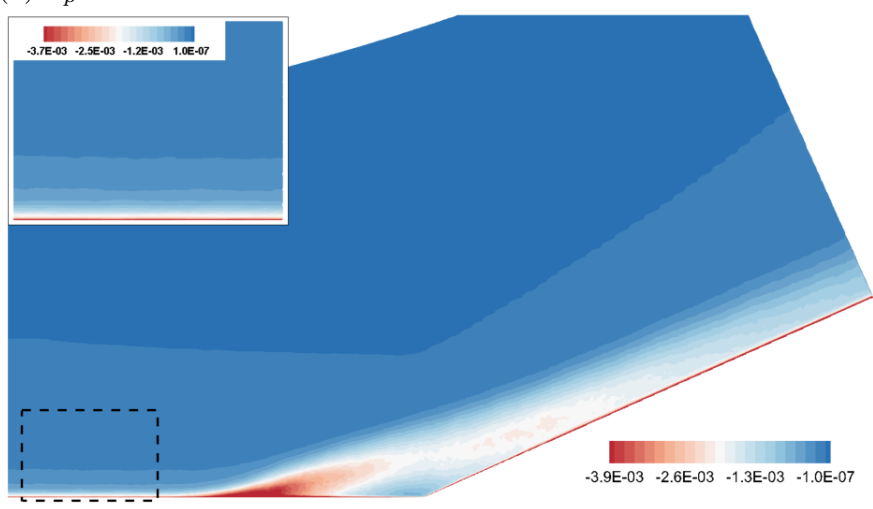

(f) $\varepsilon$

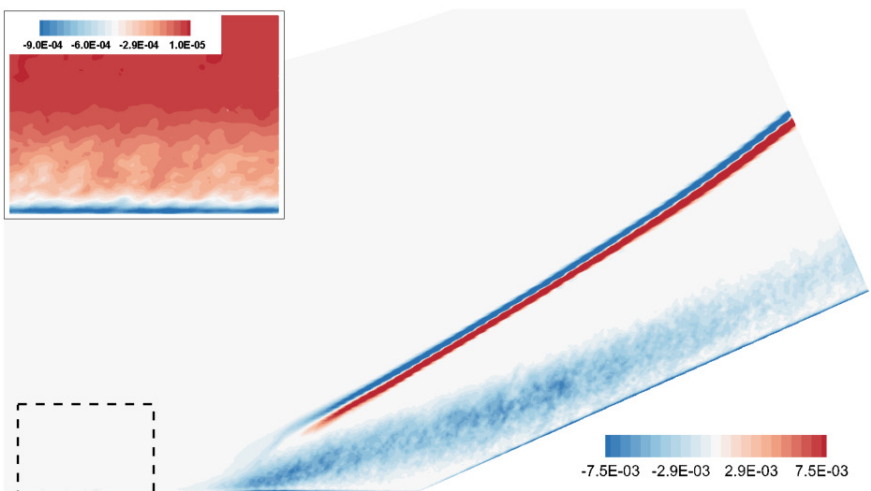

(h) $\Pi_{d}$

FIG. 15. Turbulence kinetic energy budget terms for the $9^{\text {th }}$-order WENO scheme: (a) Convection $-C$, (b) Production $-\mathrm{P}$, (c) Turbulent diffusion $-\mathrm{T}_{d}$, (d) Pressure diffusion $-\mathrm{T}_{p}$, (e) Viscous diffusion $-\mathrm{D}_{v}$, (f) Turbulent dissipation $-\varepsilon$, (g) Turbulent Mass-flux $-\mathrm{M}$, and (h) Pressure strain/dilatation $-\Pi_{p}$. 
the magnitude of one term $\left(\mathrm{D}_{v}\right.$ or $\left.\mathrm{T}_{d}\right)$ peaks the other becomes zero, which likely indicates the two terms are in some way correlated. The same near-wall mechanism (between $\mathrm{T}_{d}$ and $\mathrm{D}_{v}$ ) is present also in the relaxation region after the reattachment of the free-shear layer. However, now the maximum TKE production $(\mathrm{P})$ is located a lot higher in the TBL. Thus the mechanism is likely an intrinsic characteristic of a TBL buffer region.

Turbulent diffusion, $\mathrm{T}_{d}$, sharply increases at the $\lambda$-shock foot for both positive and negative near-wall ranges as a result of the shock wave. Moreover, the SBLI is behind both positive and negative value layers gradually expanding.

In contrast to the positive value $\mathrm{T}_{d}$ layer $\left(y^{+}<4.5\right)$, the SBLI causes the negative value layer $\left(6.4<y^{+}<19\right)$ to be "ejected" of the wall. More precisely, the SBLI causes the undisturbed TBL to split at the boundary between the viscous sublayer and the buffer layer into two separate flows. The TBL above the viscous layer is first "uplifted" and directed away from the wall at the $\lambda$-shock foot. It then travels over the separation bubble resulting in the developing free-shear layer.

The SBLI additionally leads to the formation of a new distinct outer positive $\mathrm{T}_{d}$ layer at the $\lambda$-shock foot, just above the negative value region. A significant portion of the produced TKE transported towards the outer boundary layer edge as a result of two $\mathrm{T}_{d}$ processes:

- Separation of the negative value region contained in the buffer region from the positive value layer contained within the viscous sublayer.

- Transfer of TKE from the negative layer to the outer positive layer in the free-shear layer.

Along with the oblique shock wave in Fig. 15(d), the pressure diffusion, $\mathrm{T}_{p}$, is over an order of magnitude larger than the turbulent diffusion, $\mathrm{T}_{d}$. In contrast, the opposite holds in the separated and re-attached TBL. Moreover, $\mathrm{T}_{p}$ attains a substantially large positive value in the near-wall region below both the $\lambda$-shock foot and the re-attached TBL and is the primary mechanism of TKE transport towards the inner layer. Both turbulent and pressure diffusion are equal in magnitude, but opposite in sign, only within a narrow region at the $\lambda$ shock foot just above the sonic line. $\mathrm{T}_{p}$ weakens in the vicinity of the compression corner, particularly around the centre of the separation bubble.

The viscous diffusion $\mathrm{D}_{v}$ (Fig. 15(e)) attains a relatively large magnitude in the undisturbed TBL near to the wall. In the undisturbed TBL at $x=-8 \delta_{0}, \mathrm{D}_{v}$ comprises of three separate layers. The first layer is within the viscous sublayer $\left(y^{+}<3.7\right)$ and causes an increase in TKE $(\tilde{k})$. The second layer spans $3.7<y^{+}<15$, occupying the upper region of the viscous sublayer and lower part of the buffer layer, and causes a decrease in $\tilde{k}$. The third region spans $15<y^{+}<46$ and causes only a small increase in $\tilde{k}$. The first two layers persist downstream of the SBLI along the wall of the ramp. The third layer, however, becomes amplified by the SBLI, which also causes it to stem out towards the boundary layer edge. After that, it gradually begins to decay as the TBL returns to an equilibrium state in the relaxation region. In contrast to $\mathrm{T}_{d}$, the SBLI ejects only a small fraction of the second layer, since the second layer of $\mathrm{D}_{v}$ is much nearer to the wall. The third layer in the redeveloping TBL (relaxation region) cancels out with the negative layer.

The reduction in $\tilde{k}$ caused by $\mathrm{D}_{v}$ in the second layer preSBLI is correlated to the location of maximum TKE production (P, Fig. 15(b)). It suggests that $\mathrm{D}_{v}$ "spreads" $\tilde{k}$ outwards, towards both the inner (near-wall) and outer (edge) TBL regions. The influx of $\tilde{k}$ received in the first layer results in the sharp increase in $\mathrm{D}_{v}$ in the viscous sublayer. The viscous sublayer region thus acts like a TKE sink, receiving $\tilde{k}$ using $\mathrm{D}_{v}$ and dissipating it via $\varepsilon$. This mechanism applies along the entire length of the no-slip wall, pre- to post-SBLI.

The order of magnitude difference between the third layer and the first two in the undisturbed TBL is a consequence of the much stronger viscous effects present in the "lower" nearwall region (viscous sublayer and lower buffer region) compared to the "upper" near-wall region (upper buffer region).

Similar to the viscous diffusion term, $\mathrm{D}_{v}$, the TKE dissipation is prevalent in the near-wall region along the entire length of the wall boundary (Fig. 15(f)). In the undisturbed TBL, the dissipation reaches a maximum value at $y^{+} \approx 0.9$, which indicates grids with a $y^{+}>1$ will not accurately resolve this location.

The TKE dissipation, $\varepsilon$, attains its largest magnitude at the $\lambda$-shock foot (or foot of the separated free-shear layer), as similarly reported in the previous numerical studies of $\mathrm{Li}$ et al. ${ }^{19}$ and Tong et al. ${ }^{24}$. It then weakens above the separation bubble but begins to increase again as the free-shear layer re-attaches in the relaxation region. Both of the areas mentioned above either strongly correlate or are near regions of large TKE production $(\mathrm{P})$.

Along the ramp wall, the dissipation consumes TKE mostly transferred there utilizing turbulent diffusion, $\mathrm{T}_{d}$, rather than viscous diffusion $\mathrm{D}_{v}$. Overall, however, the former plays a more prominent role in strongly turbulent regions, while the latter in the near-wall areas. Figure 15(c) shows the diffusion of $\tilde{k}$ due to $\mathrm{T}_{d}$, indicated by the negative value (blue coloured regions), at both of the high TKE production regions (Fig. 15(b)).

In the mass-flux budget term (M), $a_{i} \equiv-\overline{u_{i}^{\prime \prime}} \equiv \overline{\rho^{\prime} u_{i}^{\prime} / \bar{\rho}}$ is the turbulent mass-flux velocity, which represents the coupling of density and velocity fluctuations. It only appears in the case of compressible or variable density turbulence and is the dominant form of advection in buoyancy-driven turbulence such as the Rayleigh-Taylor instability $\left(a_{i} \approx \tilde{u}_{i}\right)$.

It can be shown than $a_{i} \equiv \tilde{u}_{i}-\bar{u}_{i}$, which for a turbulent flow leads to the following two cases: (i) if $a_{i}=0$ then $\tilde{u}=\vec{u}$ so $\rho^{\prime}=0$, or (ii) $\operatorname{sign}\left(a_{i}\right)=\operatorname{sign}\left(\tilde{u}_{i}-\bar{u}_{i}\right)$. The first condition clearly proves that $a_{i}$ (and hence $\mathrm{M}$ ) is non-zero only in the case of compressible or variable density turbulence. The second condition alludes to the origin and nature of the turbulent mass-flux velocity; it forms when there is a difference between the mass-averaged and ensemble mean velocities, which only occurs when both the density and velocity fluctuations exist and are also not uncorrelated.

In turn, we can interpret the mass-flux budget term (M) as 
the correlation between the turbulent mass-flux velocity $\left(a_{i}\right)$ and the mean-flow acceleration caused by the resulting net gradient of the pressure and viscous stresses. Vanishing $M$ may imply any of the following three conditions:

- $a_{i}$ is zero;

- the mean-flow acceleration is zero (viscous stress and pressure surface gradients balance);

- $a_{i}$ and pressure or viscous stresses gradients are uncorrelated, i.e. are random and thus converges to zero with increasing sample size.

Retrospectively, a positive correlation $(\mathrm{M}>0)$ implies that the net force gradient drives and amplifies the corresponding mass-flux velocity (similarly to the mean-flow velocity) thus increasing TKE $(\tilde{k})$, while a negative correlation (anticorrelation) the opposite.

In the undisturbed TBL (Fig. 15(g)), $\mathrm{M}$ is found to be positive and peak at $y^{+} \approx 8$ in the buffer region. In the viscous sublayer the wall-normal component of the shearstresses gradient largely dictate the mass-flux budget term; typically $\partial \tau_{i 2} / \partial y<<0$ while the gradients of mean pressure $\left(\partial \bar{p} / \partial x_{j}\right)$ and those in the in $x$-direction of the viscous stress $\left(\partial \tau_{i 1} / \partial x\right)$ are considered negligible. $\mathrm{M}$ is negative in the viscous sub-layer most likely due to the anti-correlation of the density and velocity fluctuations, i.e. $\overline{u_{i}^{\prime \prime}} \equiv-\overline{\rho^{\prime} u_{i}^{\prime}}>0$.

The proximity to the viscous sublayer (intense viscous stresses), peak TKE production (large velocity fluctuations) and weak pressure gradient across a TBL $\left(\left|\partial p / \partial x_{i}\right| \approx 0\right)$, should imply the magnitude of $\mathrm{M}$ is large. Even so, the contribution of the turbulent mass-flux budget term (M) remains relatively low, as its peak value is an order of magnitude below several other TKE budget terms, e.g. $\mathrm{P}, \mathrm{T}_{d}, \mathrm{D}_{v}$ and $\varepsilon$. Though $\mathrm{M}$ is negative in the viscous sublayer $\left(y^{+}<1.2\right)$, it is $\sim 15$ times smaller than in the buffer region. Therefore, the contribution of the turbulent mass-fluxes is relatively low, even in the case of a supersonic TBL.

The unusual " $S$ "-like distribution of the mass-flux term (M) at the SBLI foot is a consequence of its two constituent terms. The viscous stresses are the primary contributor in the nearwall region at the leading edge of the separation bubble. In contrast, the pressure gradient $(\nabla p)$ becomes the dominant mechanism away from the wall at the $\lambda$-shock foot. In both cases, large values are indicative of strong compressibility effects (density fluctuations) in the vicinity of the SBLI. The contribution of $\mathrm{M}$ to the TKE $(\tilde{k})$ is approximately an order of magnitude less than that of the production term $\mathrm{P}$.

In the relaxation region, $M$ remains positive-valued in the mixing layer as well as subsequent re-attached TBL, though its magnitude remains an order of magnitude below other terms. Nonetheless, the turbulent mass-flux (M) is the only TKE budget term to take a (negative) value between the freeshear layer and oblique shock wave, where the mean pressure isobar level $\bar{p} / p_{\infty}=3$ is present. Density fluctuations attributed to the secondary compression waves (shocklets) are likely the cause, as evident by the density-gradient contour plot shown in Fig. 8(c).
Figure $15(\mathrm{~h})$ plots the pressure-dilatation term $\left(\Pi_{p}\right)$, commonly assumed to be negligible in many turbulence models. Indeed, $\Pi_{p}$ is weak across most of the undisturbed supersonic TBL height, apart from the vicinity of the isothermal wall, where it strengthens considerably due to the strong anticorrelation between the pressure fluctuation and velocity fluctuation gradient. The influence on $\Pi_{p}$ of the imposed heattransfer condition at the wall is uncertain.

Moreover, $\Pi_{p}$ abruptly strengthens at the $\lambda$-shock foot, as well as at the re-attachment of the free-shear layer. Both locations also closely correspond to abrupt increases in P. However, it is unclear whether the two are correlated. Similarly, though, both terms gradually weaken shortly after each peak. In the analysis of the full Reynolds-stress tensor budget terms for an impinging shockwave/boundary-layer interaction, Vyas, Yoder, and Gaitonde ${ }^{86}$ showed that the pressure diffusion term $\left(\Pi_{p}\right)$ is small but non-zero in the normal stress budgets. However, it played a crucial role in the primary shear stress budget. The construction of the governing equation for TKE (Eq. (40)) takes into account the trace of the Reynolds-stress tensor, i.e. the normal Reynolds-stress components only. Nonetheless, the above results generally suggest that in certain regions, $\Pi_{p}$ becomes significant enough to be considered.

$\Pi_{p}$ becomes negative when the sign of the pressure fluctuation and the density-weighted (Favre) velocity fluctuation gradient are opposite, e.g. compression due to a velocity fluctuation $\left(\partial u_{i}^{\prime \prime} / \partial x_{i}<0\right)$ is accompanied by a pressure compression $\left(p^{\prime}>0\right)$. We can interpret this term as a correlation between the divergence of the velocity fluctuation and the pressure change (fluctuation). If the two terms are anti-correlated, as here, then $\nabla u^{\prime \prime}$ does work by causing a change in pressure, thus dampening velocity fluctuations; TKE is absorbed due to work done by the velocity fluctuations to compress or expand the fluid. If on the other hand, the two terms are positively correlated, then instead the pressure will do work to amplify the velocity fluctuations. Thus, the predominant negative values of $\Pi_{p}$ found at the SBLI and the free-shear layer is indicative of the substantial velocity fluctuations present, which largely determine and drive the pressure fluctuations.

At and after the SBLI, the results suggest that only the turbulent transport $\left(\mathrm{T}_{d}\right)$ and advection $(\mathrm{C})$ terms play a significant role in balancing turbulent production and dissipation. Post-SBLI in the recovery region, all of the TKE budget terms begin to slowly evolve towards the former equilibrium state in the undisturbed TBL.

Before concluding the analysis, it is worth cautioning that the adiabatic recovery at the wall according to Eq. (29) is approximately $317 \mathrm{~K}$, which is 10 degrees Kelvin higher than the prescribed wall temperature in the current simulations. The isothermal wall (labeled as cold wall) has a temperature lower than the adiabatic recovery temperature $(307<317 \mathrm{~K})$. Imposing a different heat-flux boundary condition at the no-slip wall, such as an adiabatic wall (zero heat-transfer) or hot wall $\left(T_{w}>317 \mathrm{~K}\right)$, will very likely lead to significant changes in the profile and the magnitude of the turbulence budget terms. 


\section{CONCLUSIONS}

We examined the performance of the WENO implementation. We showed that implementing a "normalization" technique of the WENO candidate stencils further reduces the numerical dissipation of the scheme and thus improve the accuracy of the results, particularly for under-resolved grids. For high-order numerical schemes, such as the $11^{\text {th }}$-order WENO scheme examined in $\S$ III D, the stencil spans a large portion of the local surrounding flowfield. The stencil extent is further exaggerated on a coarser mesh, and consequently, the values can differ more significantly. Normalizing the stencil values such that there is the positive real number range, i.e. a value of candidate stencil $\in \mathbb{R}_{>0}$, also prevents negative value smoothness indicators. Specifically, the stencil values are re-scaled such that the most substantial value contained is always equal to one, ensuring Galilean invariance of the scheme (though not proven here). Lastly, in the case of only small jumps across the stencils relative to the minimum absolute value, subtracting the latter ensures the former are sufficiently amplified and "identified" by the smoothness indicators.

For the case of a shockwave (supersonic) turbulent boundary layer interaction (SBLI), the order of the scheme has a significant effect on the accuracy of the DNS results obtained. High-order methods can resolve smaller turbulent structures in the flow. Consequently, the energy entering the turbulent cascade process at the inertial scale can be distributed across a broader turbulence kinetic energy spectrum range. The higher energy content present at the resolved (large) scales in lowerorder schemes causes the recirculation bubble to shift further downstream, in contrast to the experimental and numerical (DNS) data.

Using low-Mach corrections in the subsonic region of the TBL and recirculation bubble has a negligible effect on the accuracy of the low-order schemes. The above result suggests that supersonic TBL and SBLI are primarily dominated by the processes and resolved turbulence in the supersonic regions of the flow, at least for the conditions considered here $\left(M_{\infty}=3\right)$.

We demonstrated that using high-order high-resolution methods and reasonably fine grids, DNS provides accurate estimations of several key flow metrics, which are in good agreement with reference DNS data and experimental measurements. In $\S \mathrm{V} B$ we reason that the low-frequency oscillation exhibited by the shockwave likely occurs due to interchange between the foot of the $\lambda$-shockwave and the reattachment region of the detached free-shear (mixing) layer. The incoming undisturbed TBL gradually pushes the recirculation bubble downstream and with it the shockwave too. The above flow development causes the detached shear layer to impinge onto the ramp sooner, steadily rising the pressure close to the ramp corner. The pressure rise travels upstream via the subsonic region of the TBL and separation bubble and eventually becomes sufficiently large to "push" the $\lambda$-shock foot back upstream again. The time taken for an acoustic (pressure) wave to travel back and forth said distance below the sonic line corresponds closely to that of the low-frequency motion of the shockwave. Then, as the pressure relaxes, the process is repeated, leading to the low-frequency oscillation of the oblique shock observed in SBLI.

Although lower-order schemes are inaccurate even for relatively fine grids, the Reynolds stress anisotropy componentality is consistent across all numerical schemes ( $\S \mathrm{V} C)$. The componentality of the re-attached TBL does not reach an equilibrium state post-SBLI, at least for the length of the ramp considered here; it differs to that of the former "undisturbed" state across the entire height of the turbulent boundary layer.

At the "undisturbed" TBL, several of the turbulence kinetic energy budget terms examined in $\S \mathrm{V} D$ were found to change rapidly near the wall and below the buffer layer, with the viscous diffusion $\left(\mathrm{D}_{v}\right)$ in particular peaking below a $y^{+}$value of one, at just $y^{+} \approx 0.87$.

Both the Reynolds stress anisotropy componentality and turbulence kinetic energy budget terms provided little information in unravelling the cause behind the low-frequency oscillation of the SBLI shockwave. However, the production (P) and dissipation $(\varepsilon)$ TKE budget terms, as well as the compressible turbulence mass-flux $(\mathrm{M})$ and pressure-strain $\left(\Pi_{p}\right)$ terms, are found to strengthen at the separation and reattachment points but weaken in-between above the recirculation bubble. This may allude to a possible interaction mechanism between the separation and reattachment points, though the mass-flux (M) and pressure-strain $\left(\Pi_{p}\right)$ terms are about two to three orders of magnitude weaker. The mass flux, however, displayed several distinct, unusual features:

1. An " $S$ "-like shape at the foot of the $\lambda$-shock.

2. A negative value region in the upper portion of the detached free-shear layer just above the recirculation bubble.

3. Another (negative value region) where the secondary compression waves, "shocklets", form and are produced by the re-attaching shear layer on to the ramp wall.

The results of this study provide numerical evidence of the accuracy of high-resolution and high-order methods in supersonic SBLI and acoustics. Furthermore, they shed light onto the physics of supersonic SBLI. Further investigation of the above, in different hypersonic flow regimes, is the subject of future research.

\section{ACKNOWLEDGMENTS}

This material is based upon work supported by the Air Force Office of Scientific Research under awards number FA9550-19-1-7018 and FA9550-14-1-0224. The U.S. Government is authorized to reproduce and distribute reprints for Governmental purpose notwithstanding any copyright notation thereon.

Results were obtained using the ARCHIE-WeSt High Performance Computer (www.archie-west.ac.uk) based at the University of Strathclyde. 


\section{DATA AVAILABILITY}

The data that support the findings of this study are available on request from the corresponding author.The data are not publicly available due to the funding body restrictions.

\section{REFERENCES}

${ }^{1}$ K. Ritos, D. Drikakis, , I. W. Kokkinakis, and S. M. Spottswood, "Computational aeroacoustics beneath high speed transitional and turbulent boundary layers," Computers and Fluids 203, 104520 (2020).

${ }^{2}$ K. Ritos, I. W. Kokkinakis, and D. Drikakis, "Performance of high-order implicit large eddy simulations," Computers \& Fluids 173, 307 - 312 (2018).

${ }^{3}$ K. Ritos, I. W. Kokkinakis, D. Drikakis, and S. M. Spottswood, "Implici large eddy simulation of acoustic loading in supersonic turbulent boundary layers," Physics of Fluids 29, 046101 (2017).

${ }^{4}$ P. L. Ardonceau, "The structure of turbulence in a supersonic shockwave/boundary-layer interaction," AIAA Journal 22, 1254-1262 (1984).

${ }^{5}$ D. S. Dolling and C. T. Or, "Unsteadiness of the shock wave structure in attached and separated compression ramp flows," Experiments in Fluids 3 $24-32$ (1985).

${ }^{6}$ A. J. Smits and K.-C. Muck, "Experimental study of three shock wave/turbulent boundary layer interactions," Journal of Fluid Mechanics 182, 291-314 (1987).

${ }^{7}$ S. J. Beresh, N. T. Clemens, and D. S. Dolling, "Relationship between upstream turbulent boundary-layer velocity fluctuations and separation shock unsteadiness," AIAA Journal 40, 2412-2422 (2002).

${ }^{8}$ P. Bookey, C. Wyckham, A. Smits, and P. Martin, "New experimental data of STBLI at DNS/LES accessible Reynolds numbers," in 43rd AIAA Aerospace Sciences Meeting and Exhibit (American Institute of Aeronautics and Astronautics, Reno, Nevada, 2005).

${ }^{9}$ B. Ganapathisubramani, N. T. Clemens, and D. S. Dolling, "Lowfrequency dynamics of shock-induced separation in a compression ramp interaction,” Journal of Fluid Mechanics 636, 397-425 (2009).

${ }^{10}$ Z. B. Riley, R. A. Perez, G. W. Bartram, S. M. Spottswood, B. P. Smarslok, and T. J. Beberniss, "Aerothermoelastic experimental design for the AEDC/VKF Tunnel C: Challenges associated with measuring the response of flexible panels in high-temperature, high-speed wind tunnels," Journal of Sound and Vibration 441, 96 - 105 (2019).

${ }^{11}$ S. M. Spottswood, T. J. Beberniss, T. G. Eason, R. A. Perez, J. M. Donbar, D. A. Ehrhardt, and Z. B. Riley, "Exploring the response of a thin, flexible panel to shock-turbulent boundary-layer interactions," Journal of Sound and Vibration 443, 74 - 89 (2019).

${ }^{12}$ G. S. Settles, T. J. Fitzpatrick, and S. M. Bogdonoff, "Detailed study of attached and separated compression corner flowfields in high Reynolds number supersonic flow," AIAA Journal 17, 579-585 (1979).

${ }^{13}$ D. S. Dolling and M. E. Erengil, "Unsteady wave structure near separation in a mach 5 compression ramp interaction," AIAA Journal 29, 728-735 (1991).

${ }^{14}$ N. A. Adams, "Direct simulation of the turbulent boundary layer along a compression ramp at $\mathrm{M}=3$ and $\operatorname{Re}_{\theta}=1685$," Journal of Fluid Mechanics 420, 47-83 (2000).

${ }^{15}$ D. P. Rizzetta and M. R. Visbal, "Application of large-eddy simulation to supersonic compression ramps," AIAA Journal 40, 1574-1581 (2002),

${ }^{16} \mathrm{M}$. Wu and M. P. Martin, "Direct numerical simulation of supersonic turbulent boundary layer over a compression ramp," AIAA Journal 45, 879 889 (2007).

${ }^{17}$ M. Wu and M. P. Martín, "Analysis of shock motion in shockwave and turbulent boundary layer interaction using direct numerical simulation data,' Journal of Fluid Mechanics 594, 71-83 (2008).

${ }^{18}$ E. Touber and N. D. Sandham, "Large-eddy simulation of low-frequency unsteadiness in a turbulent shock-induced separation bubble," Theoretica and Computational Fluid Dynamics 23, 79 - 107 (2009).

${ }^{19} \mathrm{X}$. Li, D. Fu, Y. Ma, and X. Liang, "Direct numerical simulation of shock/turbulent boundary layer interaction in a supersonic compression ramp," Science China Physics, Mechanics and Astronomy 53, 1651 - 1658 (2010).

${ }^{20}$ S. Priebe and M. P. Martín, "Low-frequency unsteadiness in shock waveturbulent boundary layer interaction," Journal of Fluid Mechanics 699, 149 (2012).

${ }^{21}$ B. Morgan, K. Duraisamy, N. Nguyen, S. Kawai, and S. K. Lele, "Flow physics and RANS modelling of oblique shock/turbulent boundary layer interaction," Journal of Fluid Mechanics 729, 231-284 (2013).

${ }^{22}$ S. Priebe, J. H. Tu, C. W. Rowley, and M. P. Martín, "Low-frequency dynamics in a shock-induced separated flow," Journal of Fluid Mechanics 807, 441-477 (2016).

${ }^{23}$ M. P. Martin, S. Priebe, and C. M. Helm, "Upstream and downstream influence on STBLI instability," in 46th AIAA Fluid Dynamics Conference (American Institute of Aeronautics and Astronautics, 2016).

${ }^{24} \mathrm{~F}$. Tong, C. Yu, Z. Tang, and X. Li, "Numerical studies of shock wave interactions with a supersonic turbulent boundary layer in compression corner: Turning angle effects," Computers \& Fluids 149, 56 - 69 (2017).

${ }^{25}$ K. M. Porter and J. Poggie, "Turbulence structure and large-scale unsteadiness in shock-wave / boundary layer interaction," in 55th AIAA Aerospace Sciences Meeting (American Institute of Aeronautics and Astronautics, 2017).

${ }^{26} \mathrm{~J}$. Poggie and K. M. Porter, "Flow structure and unsteadiness in a highly confined shock-wave-boundary-layer interaction," Phys. Rev. Fluids 4, 024602 (2019).

${ }^{27}$ K. M. Porter and J. Poggie, "Selective upstream influence on the unsteadiness of a separated turbulent compression ramp flow," Physics of Fluids 31, 016104 (2019).

${ }^{28}$ D. S. Dolling, "Fifty years of shock-wave/boundary-layer interaction research: What next?" AIAA Journal 39, 1517 - 1531 (2001).

${ }^{29}$ N. T. Clemens and V. Narayanaswamy, "Low-frequency unsteadiness of shock wave/turbulent boundary layer interactions," Annual Review of Fluid Mechanics 46, 469-492 (2014).

${ }^{30}$ D. V. Gaitonde, "Progress in shock wave/boundary layer interactions," Progress in Aerospace Sciences 72, 80 - 99 (2015), celebrating 60 Years of the Air Force Office of Scientific Research (AFOSR): A Review of Hypersonic Aerothermodynamics.

${ }^{31}$ Z. B. Riley, R. A. Perez, G. W. Bartram, S. M. Spottswood, B. P. Smarslok, and T. J. Beberniss, "Aerothermoelastic experimental design for the AEDC/VKF Tunnel C: Challenges associated with measuring the response of flexible panels in high-temperature, high-speed wind tunnels," Journal of Sound and Vibration 441, 96 - 105 (2019).

${ }^{32}$ S. M. Spottswood, T. J. Beberniss, T. G. Eason, R. A. Perez, J. M. Donbar, D. A. Ehrhardt, and Z. B. Riley, "Exploring the response of a thin, flexible panel to shock-turbulent boundary-layer interactions," Journal of Sound and Vibration 443, 74 - 89 (2019).

${ }^{33}$ K. Ritos, I. W. Kokkinakis, and D. Drikakis, "Physical insight into the accuracy of finely-resolved iLES in turbulent boundary layers," Computers \& Fluids 169, 309 - 316 (2018).

${ }^{34}$ K. Ritos, I. W. Kokkinakis, and D. Drikakis, "Performance of highorder implicit large eddy simulations," Computers \& Fluids 173, 307 - 312 (2018).

${ }^{35}$ K. Ritos, D. Drikakis, and I. Kokkinakis, "Acoustic loading beneath hypersonic transitional and turbulent boundary layers," Journal of Sound and Vibration 441, 50 - 62 (2019).

${ }^{36}$ B. Thornber, A. Mosedale, D. Drikakis, D. Youngs, and R. Williams, “An improved reconstruction method for compressible flows with low Mach number features," Journal of Computational Physics 227, 4873 - 4894 (2008).

${ }^{37}$ G.-S. Jiang and C.-W. Shu, "Efficient implementation of weighted ENO schemes," Journal of Computational Physics 126, 202 - 228 (1996).

${ }^{38}$ A. K. Henrick, T. D. Aslam, and J. M. Powers, "Mapped weighted essentially non-oscillatory schemes: Achieving optimal order near critical points," Journal of Computational Physics 207, 542 - 567 (2005).

${ }^{39}$ D. S. Balsara and C.-W. Shu, "Monotonicity preserving weighted essentially non-oscillatory schemes with increasingly high order of accuracy," Journal of Computational Physics 160, 405 - 452 (2000).

${ }^{40}$ E. M. Taylor, M. Wu, and M. P. Martín, "Optimization of nonlinear error for weighted essentially non-oscillatory methods in direct numerical simulations of compressible turbulence," Journal of Computational Physics 223 , 384 - 397 (2007). 
${ }^{41}$ B. Van Leer, "Towards the ultimate conservative difference scheme III. Upstream-centered finite-difference schemes for ideal compressible flow,' Journal of Computational Physics 23, 263 - 275 (1977).

${ }^{42}$ K. H. Kim and C. Kim, "Accurate, efficient and monotonic numerical methods for multi-dimensional compressible flows: Part II: Multi-dimensional limiting process," Journal of Computational Physics 208, 570 - 615 (2005).

${ }^{43}$ R. Spiteri and S. Ruuth, "A new class of optimal high-order strong-stabilitypreserving time discretization methods," SIAM Journal on Numerical Analysis 40, 469-491 (2002).

${ }^{44}$ I. Kokkinakis and D. Drikakis, "Implicit large eddy simulation of weaklycompressible turbulent channel flow," Computer Methods in Applied Mechanics and Engineering 287, 229 - 261 (2015).

${ }^{45}$ P. Tsoutsanis, I. W. Kokkinakis, L. Könözsy, D. Drikakis, R. J. Williams, and D. L. Youngs, "Comparison of structured- and unstructured-grid, compressible and incompressible methods using the vortex pairing problem," Computer Methods in Applied Mechanics and Engineering 293, 207 - 231 (2015).

${ }^{46}$ C.-W. Shu, "Numerical experiments on the accuracy of ENO and modified ENO schemes," Journal of Scientific Computing 5, 127-149 (1990).

${ }^{47}$ X.-D. Liu, S. Osher, and T. Chan, "Weighted essentially non-oscillatory schemes," Journal of Computational Physics 115, 200 - 212 (1994).

${ }^{48}$ C.-W. Shu, "Essentially non-oscillatory and weighted essentially nonoscillatory schemes for hyperbolic conservation laws," in Advanced Numerical Approximation of Nonlinear Hyperbolic Equations: Lectures given a the 2 nd Session of the Centro Internazionale Matematico Estivo (C.I.M.E. held in Cetraro, Italy, June 23-28, 1997, edited by A. Quarteroni (Springer Berlin Heidelberg, Berlin, Heidelberg, 1998) pp. 325-432.

${ }^{49} \mathrm{C}$. Shu, "High order weighted essentially nonoscillatory schemes for convection dominated problems," SIAM Review 51, 82-126 (2009).

${ }^{50}$ O. Zanotti and M. Dumbser, "Efficient conservative ADER schemes based on WENO reconstruction and space-time predictor in primitive variables,' Computational Astrophysics and Cosmology 3, 1-32 (2016).

${ }^{51}$ R. H. Nichols, R. W. Tramel, and P. G. Buning, "Evaluation of two high-order weighted essentially nonoscillatory schemes," AIAA Journal 46 , 3090-3102 (2008).

${ }^{52}$ H. Feng, C. Huang, and R. Wang, "An improved mapped weighted essentially non-oscillatory scheme," Applied Mathematics and Computation 232, 453 - 468 (2014).

${ }^{53}$ A. Harten, B. Engquist, S. Osher, and S. R. Chakravarthy, "Uniformly high order accurate essentially non-oscillatory schemes, III," Journal of Computational Physics 131, 3 - 47 (1997).

${ }^{54} \mathrm{~V}$. Titarev and E. Toro, "Finite-volume WENO schemes for threedimensional conservation laws," Journal of Computational Physics 201 238 - 260 (2004).

${ }^{55}$ E. F. Toro, M. Spruce, and W. Speares, "Restoration of the contact surface in the HLL-Riemann solver," Shock Waves 4, 25 - 34 (1994).

${ }^{56}$ E. F. Toro, Riemann Solvers and Numerical Methods for Fluid Dynamics, A Practical Introduction, 3rd ed. (Springer-Verlag Berlin Heidelberg, 2009).

${ }^{57}$ E. F. Toro, "A linearized Riemann solver for the time-dependent Euler equations of gas dynamics," Proceedings of the Royal Society of London. Series A: Mathematical and Physical Sciences 434, 683-693 (1991).

${ }^{58}$ E. F. Toro, "Direct Riemann solvers for the time-dependent Euler equations," Shock Waves 5, 75 (1995).

${ }^{59}$ S. Davis, "Simplified second-order Godunov-type methods," SIAM Journal on Scientific and Statistical Computing 9, 445-473 (1988).

${ }^{60} \mathrm{~J}$. DeBonis, "Solutions of the Taylor-Green vortex problem using highresolution explicit finite difference methods," in 51 st AIAA Aerospace Sciences Meeting including the New Horizons Forum and Aerospace Exposition (American Institute of Aeronautics and Astronautics, 2013).

${ }^{61}$ C. Bogey and C. Bailly, "A family of low dispersive and low dissipative explicit schemes for flow and noise computations," Journal of Computationa Physics 194, 194 - 214 (2004).

${ }^{62} \mathrm{Z}$. Rana, B. Thornber, and D. Drikakis, "Transverse jet injection into a supersonic turbulent cross-flow," Physics of Fluids 23, 046103 (2011).

${ }^{63}$ T. S. Lund, X. Wu, and K. D. Squires, "Generation of turbulent inflow data for spatially-developing boundary layer simulations," Journal of Computational Physics 140, 233 - 258 (1998).

${ }^{64}$ M. Klein, A. Sadiki, and J. Janicka, "A digital filter based generation of inflow data for spatially developing direct numerical or large eddy simula- tions," Journal of Computational Physics 186, 652 - 665 (2003).

${ }^{65}$ Z. A. Rana, B. Thornber, and D. Drikakis, "On the importance of generating accurate turbulent boundary condition for unsteady simulations," Journal of Turbulence 12, N35 (2011)

${ }^{66}$ T. Cebeci and A. Smith, Analysis of Turbulent Boundary Layers (Academic Press, 1974).

${ }^{67}$ J. Gaviglio, "Reynolds analogies and experimental study of heat transfer in the supersonic boundary layer," International Journal of Heat and Mass Transfer 30, 911 - 926 (1987).

${ }^{68}$ K. Ritos, D. Drikakis, and I. W. Kokkinakis, "Wall-pressure spectra models for supersonic and hypersonic turbulent boundary layers," Journal of Sound and Vibration 443, $90-108$ (2019).

${ }^{69}$ D. A. Bies, "A review of flight and wind tunnel measurements of boundary layer pressure fluctuations and induced structural response," Tech. Rep. NASA-CR-626 (NASA, 1965).

${ }^{70}$ A. V. Smol'yakov, "Calculation of the spectra of pseudosound wallpressure fluctuations in turbulent boundary layers," Acoustical Physics 46 , 342-347 (2000).

${ }^{71}$ N. J. Georgiadis, D. P. Rizzetta, and C. Fureby, "Large-eddy simulation: current capabilities, recommended practices, and future research." AIAA Journal 48, 1172-1784 (2010).

${ }^{72} \mathrm{H}$. Choi and P. Moin, "Grid-point requirements for large eddy simulation: Chapman's estimates revisited," Physics of Fluids 24, 011702 (2012).

${ }^{73}$ J. Poggie, N. J. Bisek, and R. Gosse, "Resolution effects in compressible, turbulent boundary layer simulations," Computers \& Fluids 120, 57 69 (2015).

${ }^{74}$ I. W. Kokkinakis and D. Drikakis, "Near-wall behaviour of implicit large eddy simulations," in ECCOMAS Congress 2016 - Proceedings of the 7th European Congress on Computational Methods in Applied Sciences and Engineering, Vol. 1 (National Technical University of Athens, 2016) pp. 1032-1045.

${ }^{75}$ S. Xu and M. P. Martin, “Assessment of inflow boundary conditions for compressible turbulent boundary layers," Physics of Fluids 16, 2623-2639 (2004)

${ }^{76}$ V. Kolář, "Compressibility effect in vortex identification," AIAA Journal 47, 473-475 (2009).

${ }^{77}$ V. Kolář and J. Šístek, "Corotational and compressibility aspects leading to a modification of the vortex-identification Q-criterion,” AIAA Journal 53, 2406-2410 (2015).

${ }^{78}$ L. Duan, M. M. Choudhari, and C. Zhang, "Pressure fluctuations induced by a hypersonic turbulent boundary layer,' Journal of Fluid Mechanics 804. 578-607 (2016)

${ }^{79}$ M. Bull, "Wall-pressure fluctuations beneath turbulent boundary layers: some reflections on forty years of research," Journal of Sound and Vibration 190, 299 - 315 (1996)

${ }^{80} \mathrm{M}$. Bernardini, S. Pirozzoli, and F. Grasso, "The wall pressure signature of transonic shock/boundary layer interaction," Journal of Fluid Mechanics 671, 288-312 (2011).

${ }^{81}$ W. Schneider, "Upstream propagation of unsteady disturbances in supersonic boundary layers," Journal of Fluid Mechanics 63, 465-485 (1974).

${ }^{82}$ G. S. Settles, S. M. Bogdonoff, and I. E. Vas, "Incipient separation of a supersonic turbulent boundary layer at high Reynolds numbers," AIAA Journal 14, 50-56 (1976).

${ }^{83}$ P. Bookey, C. Wyckham, and A. Smits, "Experimental investigations of Mach 3 shock-wave turbulent boundary layer interactions," in 35th AIAA Fluid Dynamics Conference and Exhibit (2005).

${ }^{84}$ S. Banerjee, R. Krahl, F. Durst, and C. Zenger, "Presentation of anisotropy properties of turbulence, invariants versus eigenvalue approaches," Journal of Turbulence 8, N32 (2007).

${ }^{85} \mathrm{M}$. Emory and G. Iaccarino, "Visualizing turbulence anisotropy in the spatial domain with componentality contours," Tech. Rep. (Center for Turbulence Research, Annual Research Briefs, Stanford University, 2014).

${ }^{86}$ M. A. Vyas, D. A. Yoder, and D. V. Gaitonde, "Reynolds-stress budgets in an impinging shock wave/boundary-layer interaction," in 2018 AIAA Aerospace Sciences Meeting (American Institute of Aeronautics and Astronautics, 2018) pp. 1-25. 


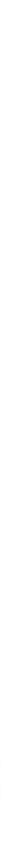

(a)

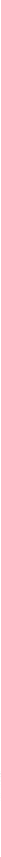

(b) 
Supersonic Flow

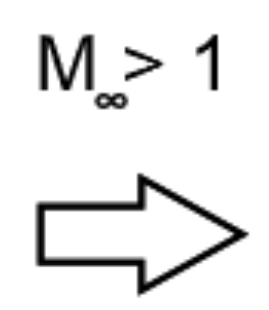

Incoming Turbulent Boundary Layer

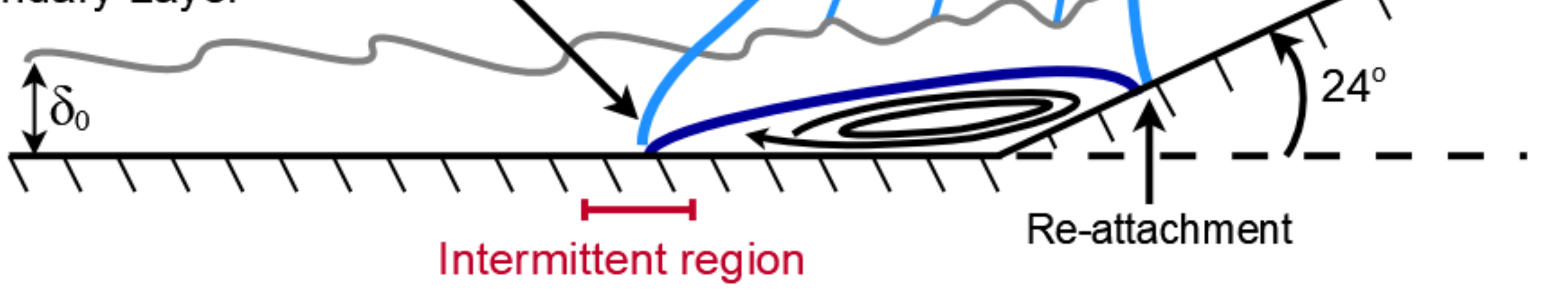




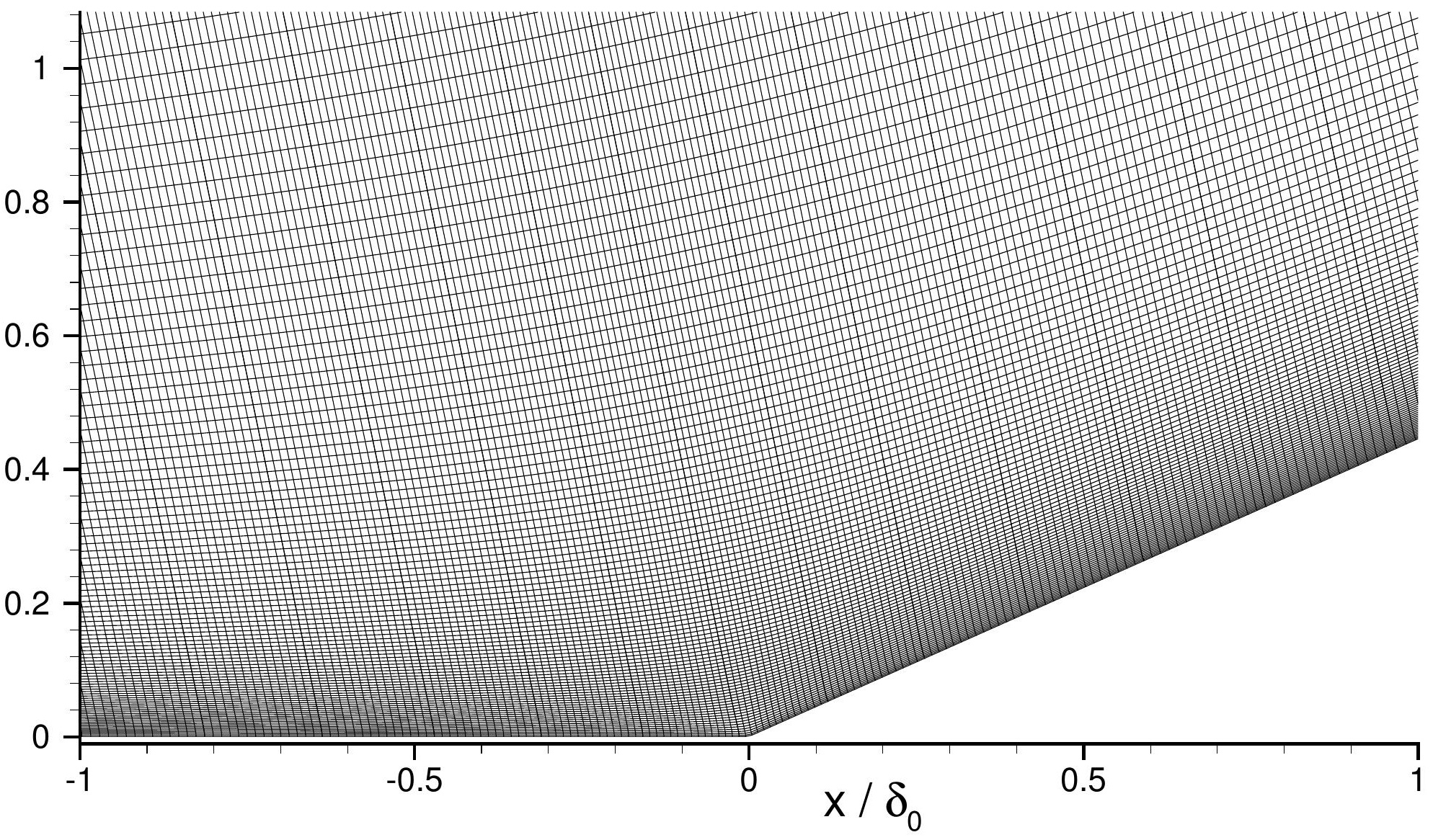




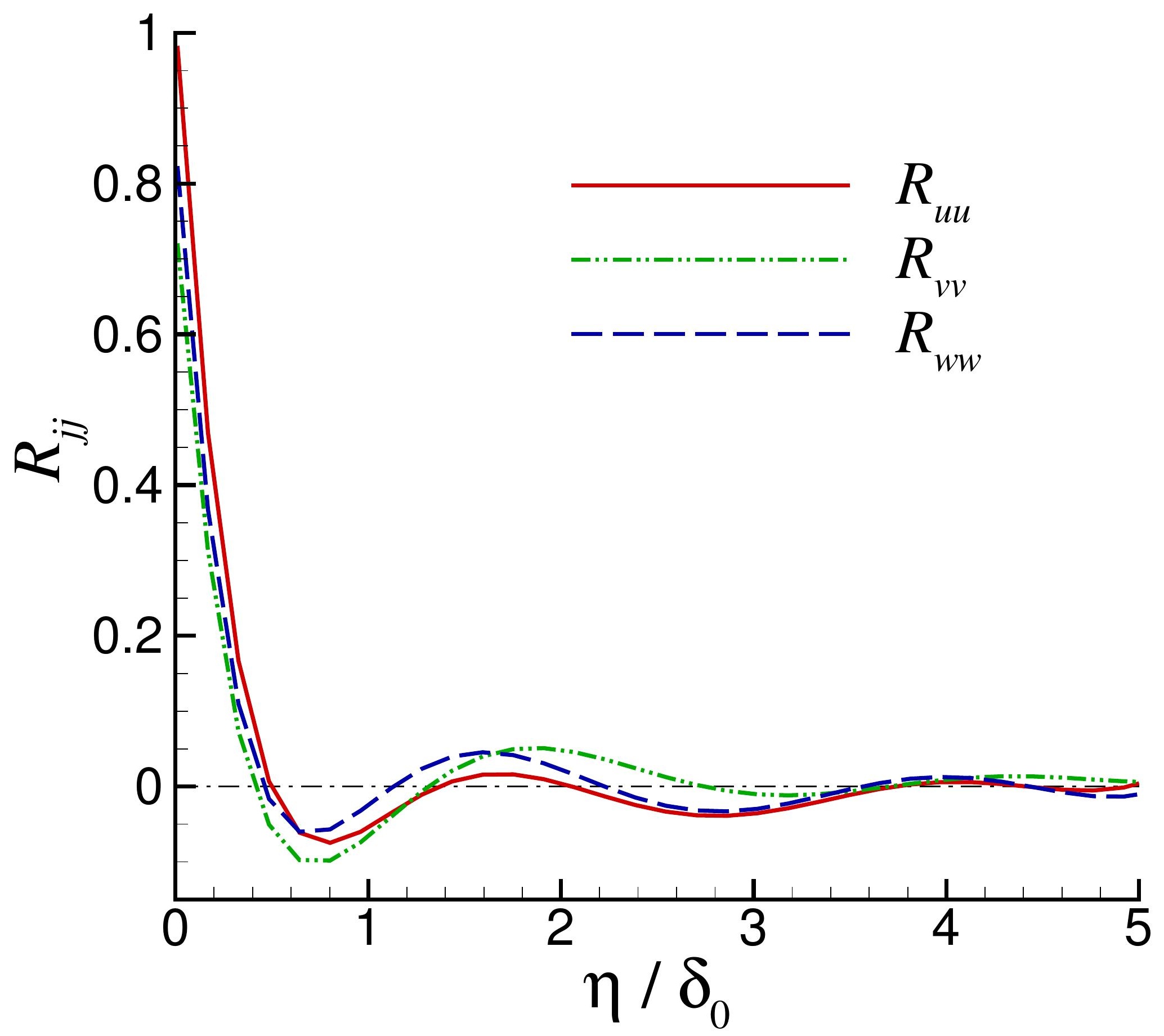




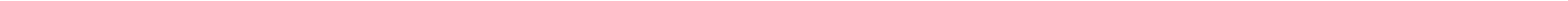




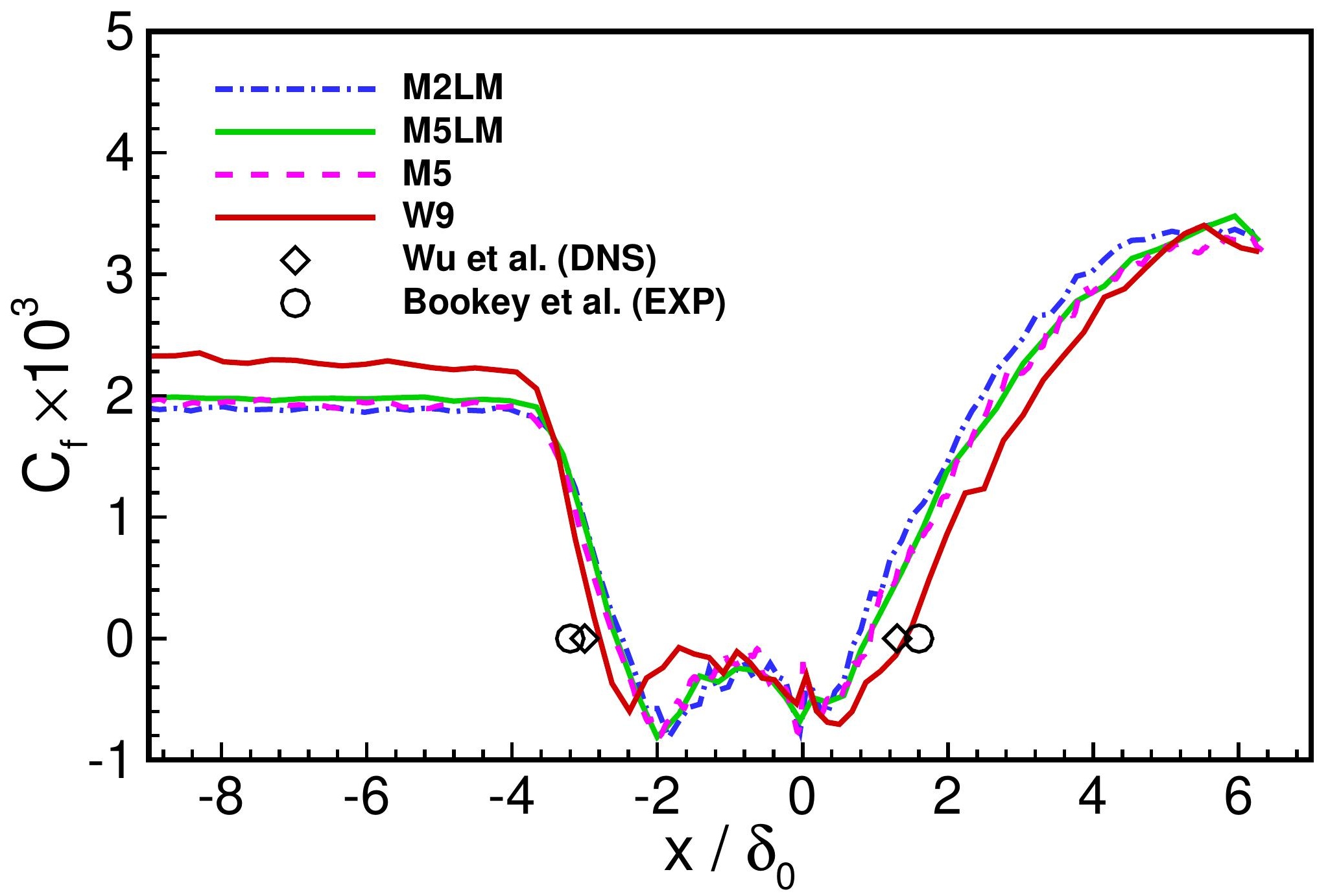




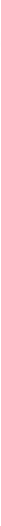

(a) $x=-8.0 \delta_{0}$

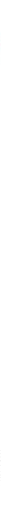

(b) $x=-1.9 \delta_{0}$

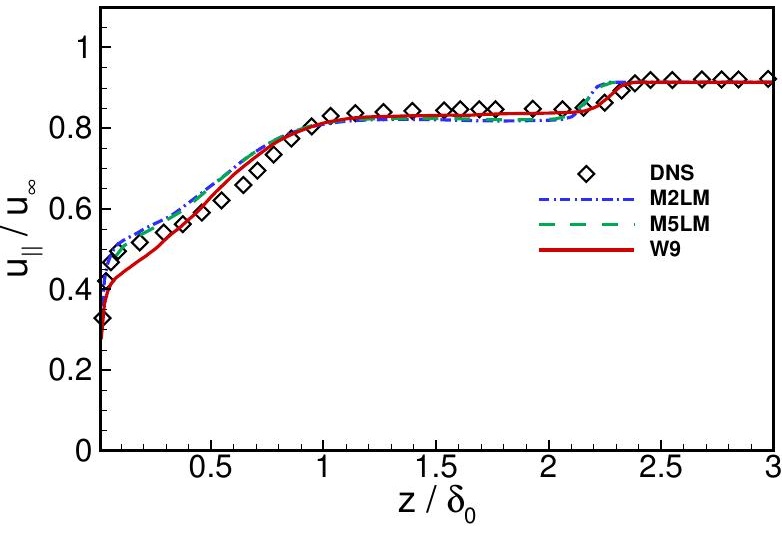

(c) $x=6.1 \delta_{0}$ 


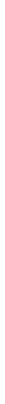

(a)
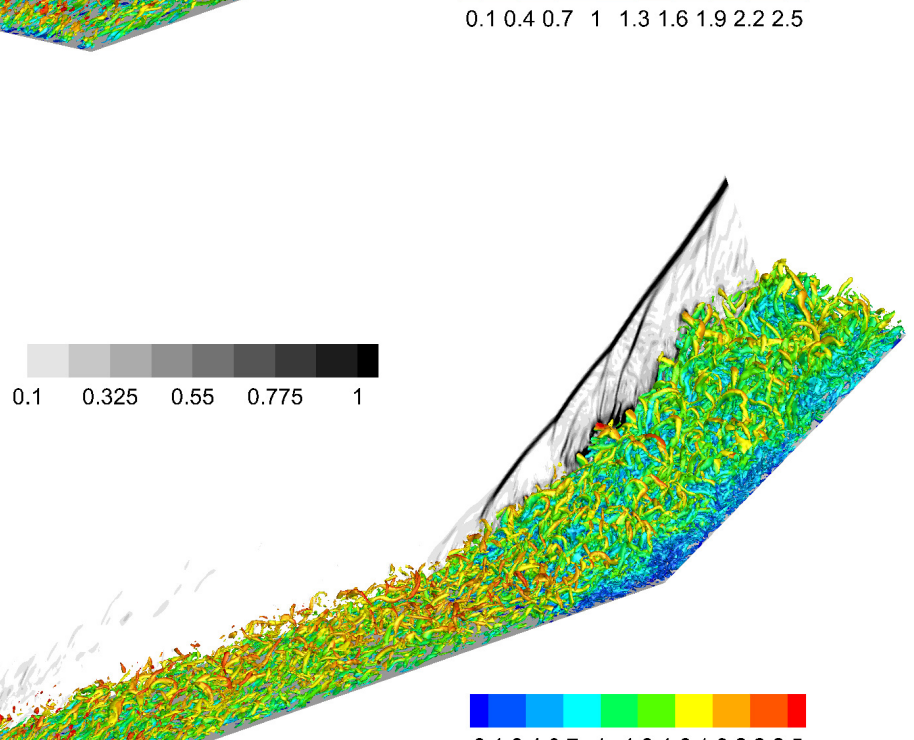

$0.10 .40 .7 \quad 1 \quad 1.31 .61 .92 .22 .5$

(b)

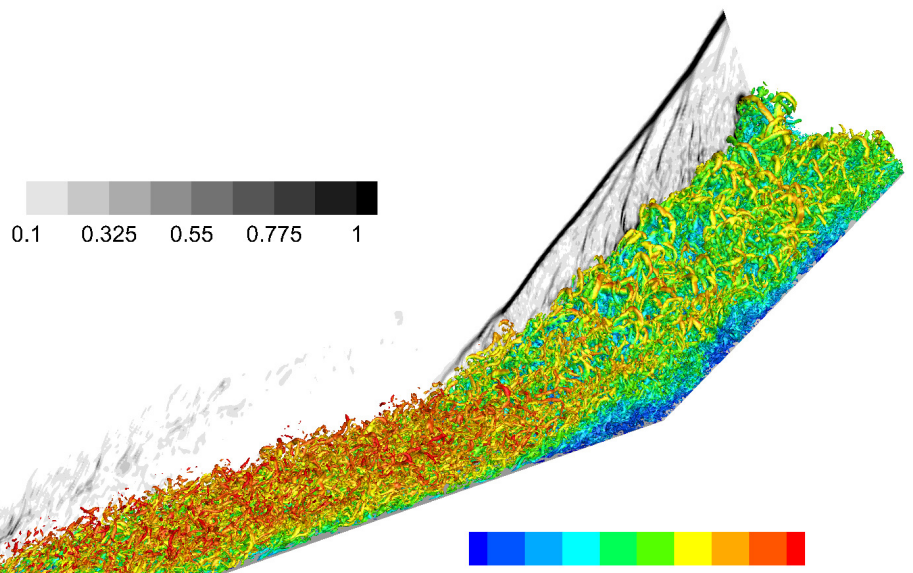

$0.10 .40 .7 \quad 1 \quad 1.31 .61 .92 .22 .5$

(c) 


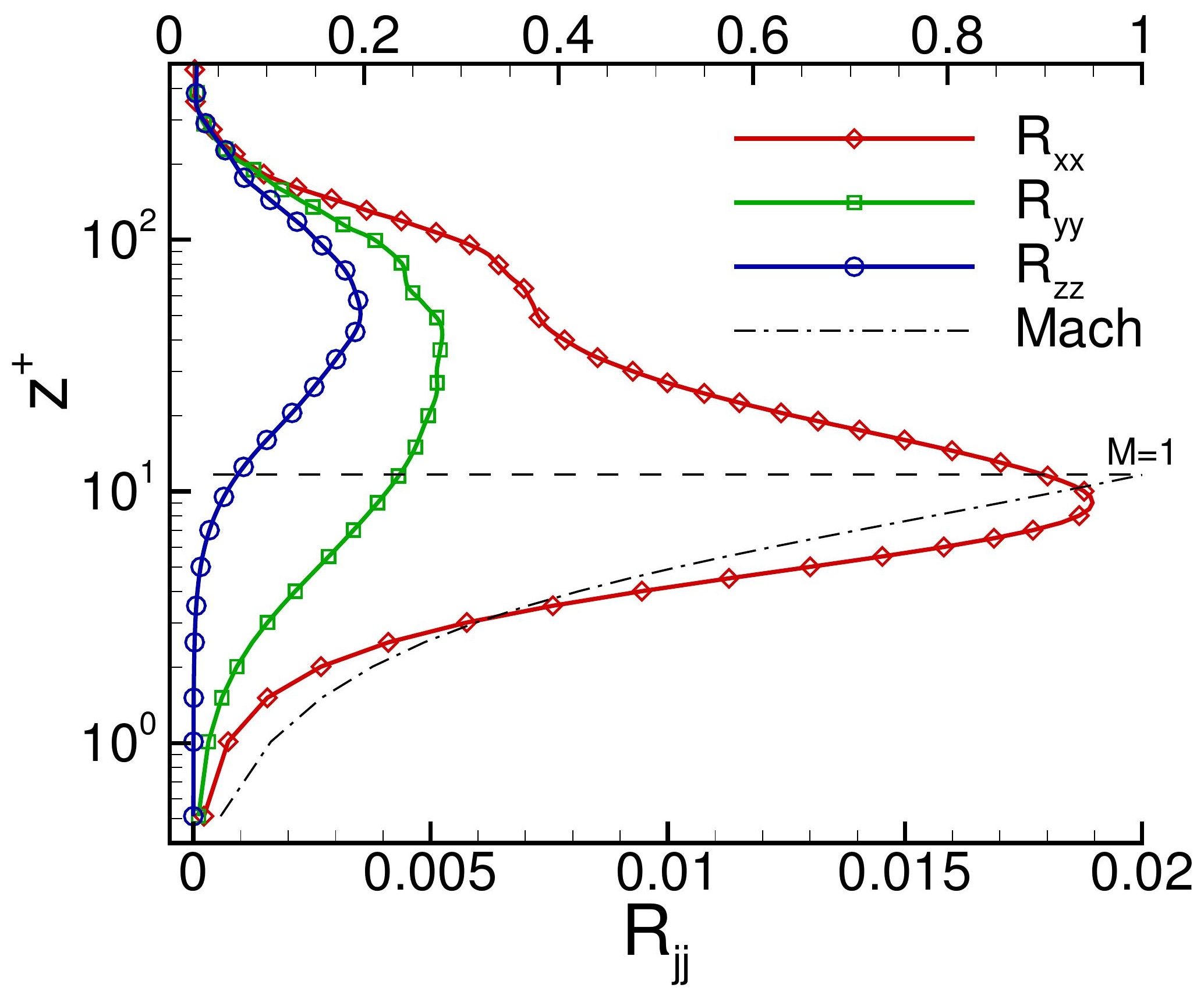




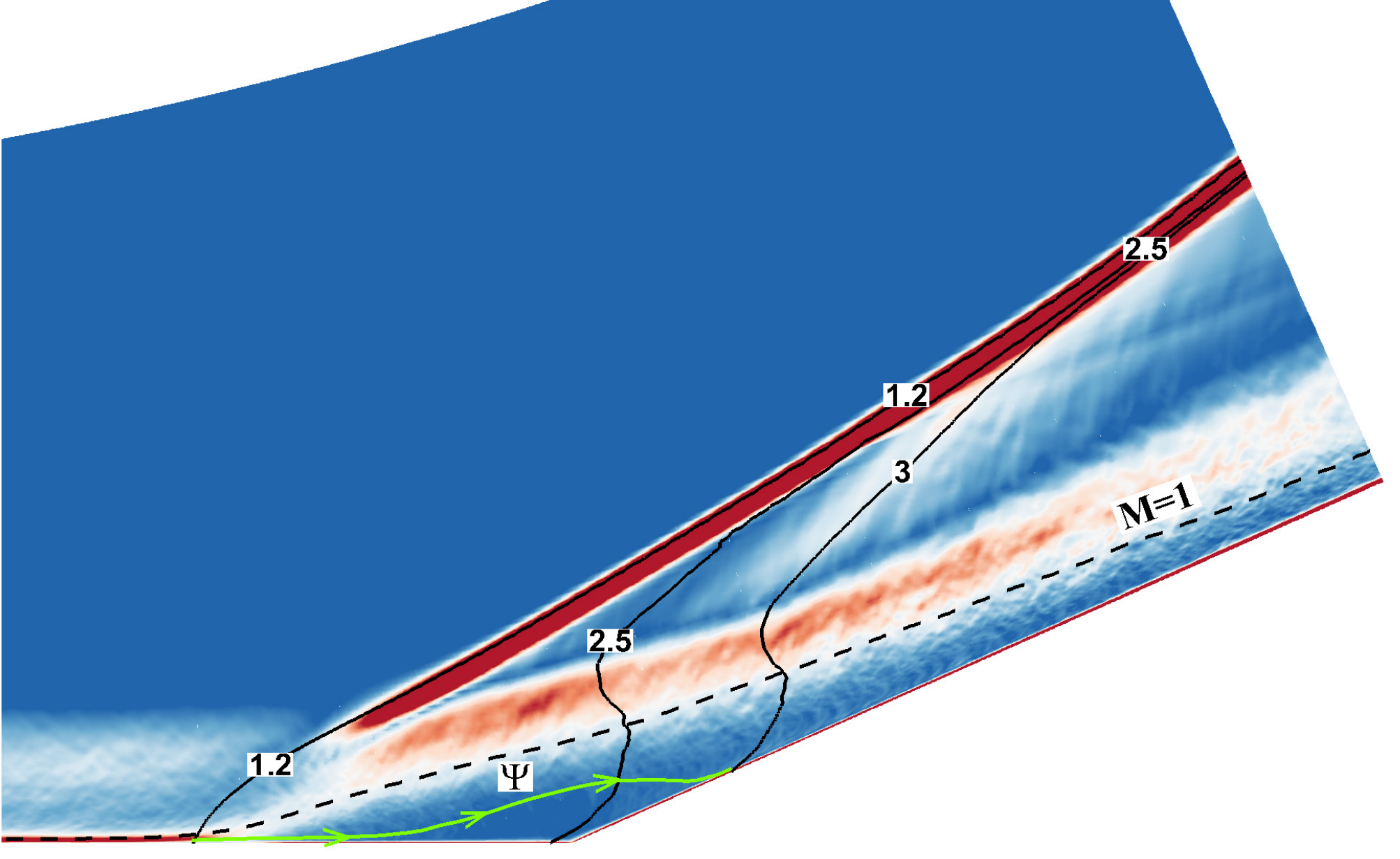




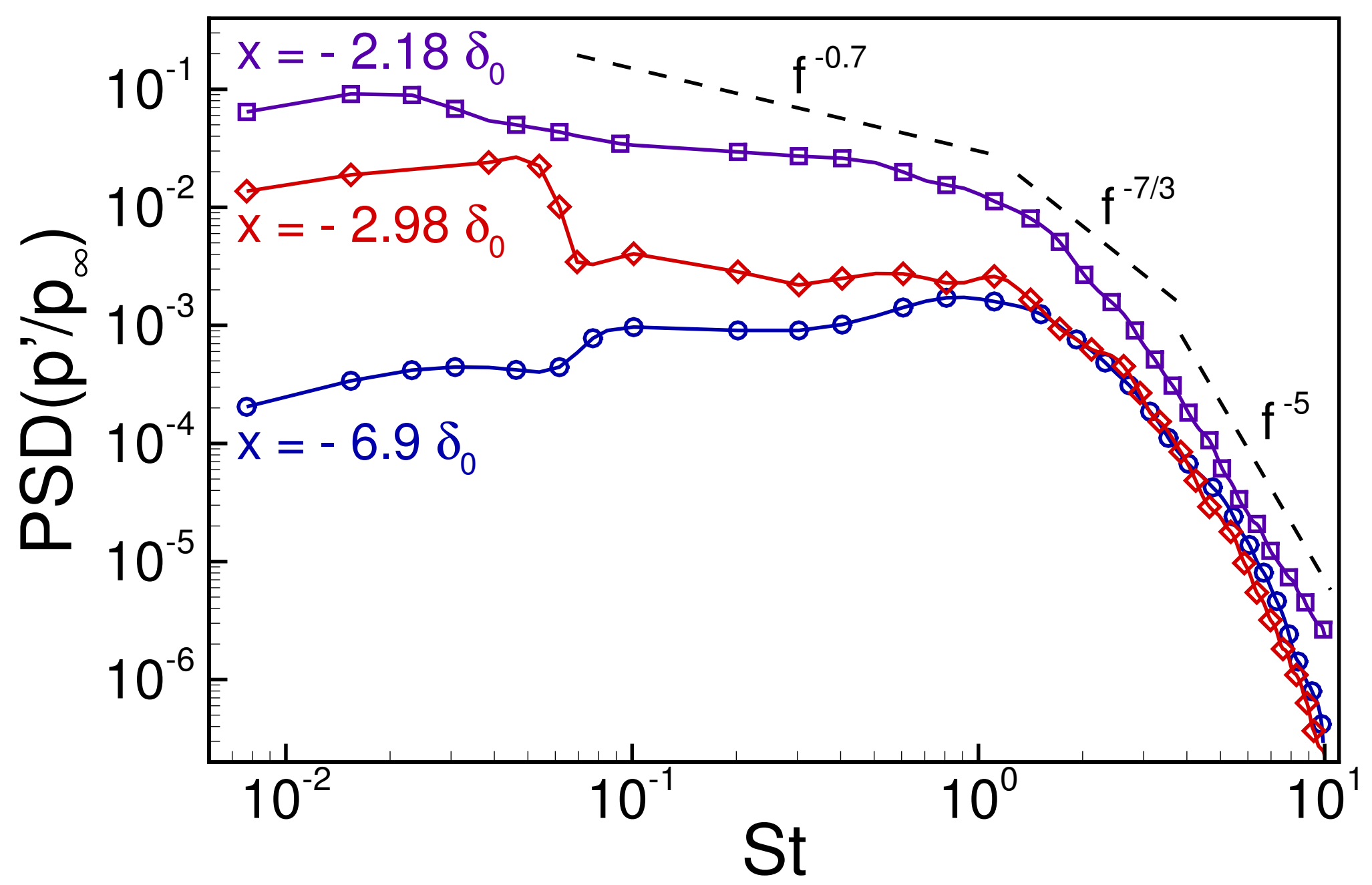




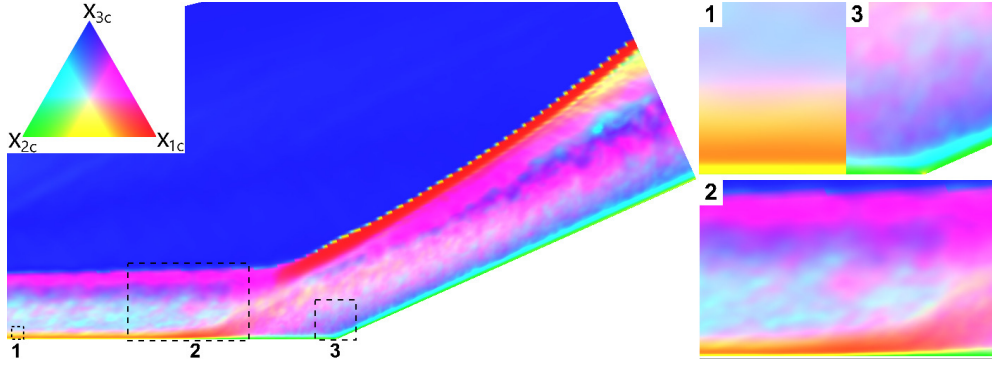

(a)

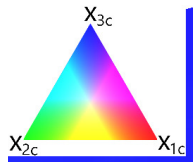

1

3
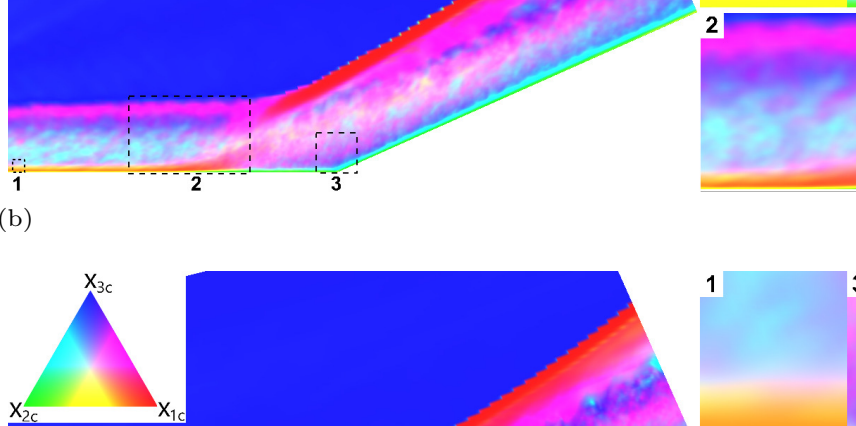

1

3

5

(b)
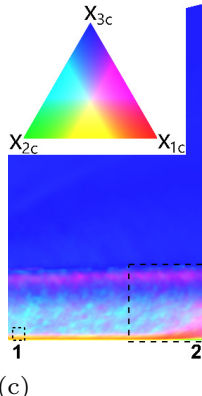

${ }_{3}$

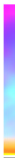

(c) 
$\mathrm{X}_{2 \mathrm{c}} \quad \mathrm{X}_{1 \mathrm{c}}$

2 
